\title{
The number density of a charged relic
}

\author{
Carola F. Berger ${ }^{1,2}$, Laura Covi ${ }^{3}$, Sabine $\mathrm{Kraml}^{4}$ \\ and Federica Palorini ${ }^{5}$
}

(1) Center for Theoretical Physics, Massachusetts Institute of Technology, Cambridge, MA 02139, USA

(2) Kavli Institute for Theoretical Physics, University of California, Santa Barbara, CA 93106-4030, USA

(3) DESY Theory Group, Notkestrasse 85, D-22603 Hamburg, Germany

(4) Laboratoire de Physique Subatomique et de Cosmologie, UJF Grenoble 1, CNRS/IN2P3, 53 Avenue des Martyrs, F-38026 Grenoble, France

(5) IPNL, Université de Lyon, Université Lyon 1, CNRS/IN2P3, 4 rue E. Fermi, F-69622 Villeurbanne, France

\begin{abstract}
We investigate scenarios in which a charged, long-lived scalar particle decouples from the primordial plasma in the Early Universe. We compute the number density at time of freeze-out considering both the cases of abelian and non-abelian interactions and including the effect of Sommerfeld enhancement at low initial velocity. We also discuss as extreme case the maximal cross section that fulfils the unitarity bound. We then compare these number densities to the exotic nuclei searches for stable relics and to the BBN bounds on unstable relics and draw conclusions for the cases of a stau or stop NLSP in supersymmetric models with a gravitino or axino LSP.
\end{abstract}




\section{Contents}

1 Introduction

2 Number density of a thermal relic

3 Annihilation cross section for a charged particle into gauge bosons 6

3.1 Abelian case . . . . . . . . . . . . . . . . . 6

3.2 Non-abelian case . . . . . . . . . . . . . . . . . . . . 6

3.3 Annihilation into $S U(N)$ gauge boson and photon . . . . . . . . . . 8

3.4 Annihilation into physical Z and $S U(N)$ gauge boson/photon . . . . . . 9

3.5 Annihilation into massless EW gauge bosons . . . . . . . . . . . . . . . . 10

3.6 Sommerfeld enhancement . . . . . . . . . . . . . . . . . . . . 10

3.7 Unitarity bound . . . . . . . . . . . . . . . . . . . . 14

3.8 Thermally averaged cross sections and velocity expansion . . . . . . . . 15

4 Results for the relic density $\quad \lcm{16}$

5 Constraints on cosmological relics $\quad \mathbf{1 8}$

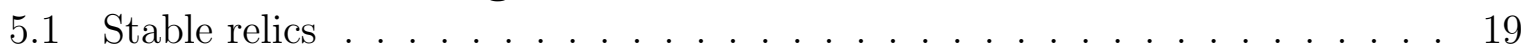

5.2 Unstable relics . . . . . . . . . . . . . . . . . . . . . . . . . . . . . 20

6 Application to the MSSM $\mathbf{2 2}$

6.1 Relic stau . . . . . . . . . . . . . . . . . . . . . . . . . . . . . . . . . . . .

6.2 Relic stop . . . . . . . . . . . . . . . . . . 26

$\begin{array}{lll}7 & \text { Conclusions } & 28\end{array}$

\begin{tabular}{ll} 
Acknowledgements & 29 \\
\hline
\end{tabular}

A Annihilation into massless $S U(N)$ gauge bosons 30

A.1 Amplitudes for the annihilation ................ . . . . . . . . . . . . . . . . .

A.2 The matrix element . . . . . . . . . . . . . . . . . . . 31

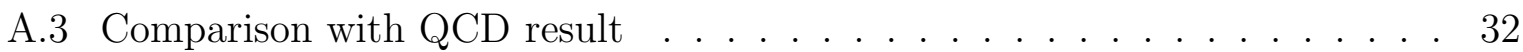

B Annihilation into $S U(2)_{L}$ gauge bosons 33

B.1 $S U(2)_{L}$ sum and total matrix element . . . . . . . . . . . . . . . . . . . . . . . . .

B.2 Annihilation into physical $W^{+} W^{-} \ldots \ldots \ldots \ldots \ldots$

B.3 Polarisation sum . . . . . . . . . . . . . . . . . 37

B.4 Symmetric part . . . . . . . . . . . . . . . . . . . . . . . . . . . . . . . . . . . .

B.5 Antisymmetric part . . . . . . . . . . . . . . . . . . . 39

B.6 Results for the cross section . . . . . . . . . . . . . . . . 41

References 


\section{Introduction}

The early Universe may have been populated by many exotic particles that, especially if charged, should have easily been in thermal equilibrium. No charged relic seems to have survived to the present day. In fact there are very strong upper bounds on the density of electromagnetically and/or colour charged particles with masses below 10-100 TeV from extensive searches for exotic nuclei [1]. The standard lore is therefore that only neutral relics may have survived until today.

However, it is possible that some unstable but very long-lived charged particle frozeout from thermal equilibrium and decayed much later to a neutral one. A typical example of this kind in supersymmetric models with R-parity conservation is the next-to-lightest supersymmetric particle (NLSP) if the LSP and Cold Dark Matter is very weakly interacting like the axino [2, 3, 4] or the gravitino [5, 6]. Recently, such candidates have attracted a lot of attention, and indeed the signal of a charged metastable NLSP at colliders would be spectacular [7, 8].

In general, strong bounds on the number density of any metastable relic with lifetime of about $1 \mathrm{~s}$ or longer are provided by Big Bang Nucleosynthesis (BBN) [9]. They come from two classes of processes: on one hand injection of very energetic photons or hadrons from decays during or after BBN adds an additional non-thermal component to the plasma and can modify the abundances of the light elements [10]; on the other hand, if the relic particle is electromagnetically charged, bound states with nuclei may arise that strongly enhance some of the nuclear rates and allow for catalysed production of e.g. ${ }^{6} \mathrm{Li}$ [11]. The bounds of the first type are very tight for lifetimes of the order of $10^{4} \mathrm{~s}$ and exclude, for instance, a neutralino NLSP with a gravitino LSP in the CMSSM [6]. An electrically charged NLSP like the $\tilde{\tau}$ can instead escape the first class of constraints in part of the parameter space, but it is excluded for long lifetimes by bound state effects [12]. In the axino LSP case, the NLSP has a shorter lifetime; the BBN bounds are hence much weaker and both, neutralino and stau, NLSP are still allowed [2].

In this paper, we investigate the most general case of a scalar charged thermal relic. We compute the number density and compare it to the bounds on exotic nuclei for stable particles and the BBN constraints for unstable ones. Similar studies have been carried out model-independently many years ago [13, 14, 15] for stable relics and we will update and improve these computations 1 We mostly consider the role of the gauge interaction for two main reasons: i) the annihilation into gauge bosons is often the dominant channel for a charged particle and ii) it depends only on very few parameters, just the mass of the particle and its charge or representation. It is also enhanced by the Sommerfeld effect [17, analogous to heavy quark production at threshold, which has previously been considered for dark matter annihilations in [18, 19, 16, 20, 21] and recently also in the context of leptogenesis in [22]. We discuss this Sommerfeld enhancement for the general abelian and non-abelian cases. Moreover, we compare the cross sections with the unitarity bound and update the unitarity limit on the mass of a stable relic.

Our main goal is to determine if it is at all possible to evade completely either the exotic nuclei bounds or the BBN ones and how strongly the particle has to interact in this case. We then apply our findings to the Minimal Supersymmetric Standard Model and discuss in more detail the cases of the stau and stop NLSP.

\footnotetext{
${ }^{1}$ Recently the case of general EW charged relics as DM was also considered in full detail [16].
} 
The paper is organised as follows. In Section 2, we briefly review the computation of the number density from thermal freeze-out. The formulae for the annihilation cross section of a charged particle into gauge bosons are given in Section 3. Here we discuss abelian and non-abelian cases, the Sommerfeld enhancement and the unitarity cross section. Moreover, we compare the thermal averages with the first order in velocity expansion. The resulting relic density is discussed in Section 4. In Section 5, we review the constraints on stable and unstable relics. These are then applied in Section 6 to the concrete examples of relic staus and stops. Section 7 finally contains our conclusions. Details on the computation of the annihilation cross section and the case of massive gauge bosons are given in the Appendices A and B.

\section{Number density of a thermal relic}

The number density of a stable or quasi-stable thermal relic is determined by its annihilation cross section. In fact the number density of a particle in a thermal bath and an expanding Universe is described by the Boltzmann equation [23, 24]:

$$
\dot{n}_{X}+3 H n_{X}=\int \frac{d p_{X}^{3}}{(2 \pi)^{3} 2 E_{X}} \mathcal{C}\left[f_{X}\right]
$$

where the dot indicates the time derivative, $\mathcal{C}$ denotes the collision integral of all processes that change the particle number and $f_{X}$ is the phase-space density for the particle $X$. For a particle with a conserved parity, like R-parity, the lowest order processes to be considered in the collision integral are just two particle scatterings, i.e. annihilations and coannihilations. If there is a lighter particle carrying the conserved parity number, $\mathcal{C}$ includes also the decay into this lighter state, but we will assume that such a decay rate is so small it can be neglected at the time of freeze-out and becomes effective only much later. Then we have effectively a two step process and we can treat freeze-out and decay separately. This is a general feature if the decay takes place via a non-renormalisable interaction and is suppressed by an intermediate or even the Planck scale (see e.g. the axino [2, 4] and gravitino cases [5, 6]).

Taking into account only the annihilation of particle and antiparticle, we can write the collision integral as [24]

$$
\mathcal{C}\left[f_{X}\right]=-\int \frac{d p_{\bar{X}}^{3}}{(2 \pi)^{3} 2 E_{\bar{X}}}\left(f_{X} f_{\bar{X}}-f_{X}^{e q} f_{\bar{X}}^{e q}\right) 4 \sqrt{\left(p_{X} \cdot p_{\bar{X}}\right)-m_{X}^{4}} \sigma_{a n n}
$$

where $\sigma_{a n n}$ denotes the unpolarised annihilation cross section of an $X \bar{X}$ pair summed over initial and final states. We are here assuming that $\mathrm{CP}$ is conserved and no asymmetry exists between $n_{X}$ and $n_{\bar{X}}$. Note that the production cross section is taken into account by the term proportional to $f_{X}^{e q} f_{\bar{X}}^{e q}$ since we are assuming that the products of the annihilation are much lighter than $X$ and are still in thermal equilibrium.

In this paper we will consider charged relics and concentrate therefore on the annihilation into gauge bosons, which is the dominant channel in most of parameter space and does depend only on the mass and charge of the relic. Note that adding more channels only increases the cross section and reduces the relic particle number density further. Instead, the inclusion of coannihilations for a charged particle does not always reduce the number density as discussed in [25]. 
We can rewrite eq. (11) by changing variable to $Y_{X}=n_{X} / s$, where $s(T)=g_{S} \frac{2 \pi^{2}}{45} T^{3}$ is the entropy density, so that the dilution due to the expansion of the universe cancels out in the ratio as long as entropy is conserved. It is also convenient to replace the time variable with $x=\frac{m_{X}}{T}$, thanks to the relation $d t=\frac{d x}{(x H)}$. We thus obtain

$$
\begin{aligned}
\frac{d Y_{X}}{d x} & =-\frac{x s(x)}{H(x) m_{X}^{2}}\langle\sigma v\rangle_{x}\left(Y_{X}^{2}-Y_{e q}^{2}\right) \\
& =-\frac{2 \pi g_{S}}{15}\left(\frac{10}{g_{\rho}}\right)^{1 / 2} \frac{M_{P}}{m_{X}}\langle\sigma v\rangle_{x}\left(Y_{X}^{2}-Y_{e q}^{2}\right) .
\end{aligned}
$$

Here we have used $H^{2}=\frac{\pi^{2}}{90} g_{\rho} \frac{T^{4}}{M_{P}^{2}}$, for $M_{P}=2.43 \times 10^{18} \mathrm{GeV}$, valid during the radiation dominated era. Moreover, we define the thermally averaged cross section as 2

$$
\langle\sigma v\rangle_{x}=\frac{1}{4 x^{4} K_{2}^{2}(x)} \int_{2 x}^{\infty} d z z^{2} \tilde{\sigma}\left(\frac{x}{z}\right) K_{1}(z)
$$

where $K_{i}(z)$ are the modified Bessel functions of order $i$, characteristic of MaxwellBoltzmann statistics (we are assuming that we can approximate Bose-Einstein statistics with Maxwell-Boltzmann statistics). In this expression the rescaled cross section $\tilde{\sigma}$ is given by the annihilation cross section averaged over initial and summed over final states and multiplied by a factor proportional to the squared Møller velocity,

$$
\tilde{\sigma}\left(\frac{m_{X}}{\sqrt{s}}\right)=\left(s-4 m_{X}^{2}\right) \sigma\left(m_{X}, s\right) .
$$

Note that in the centre-of-mass system the Møller velocity is equal to the relative velocity between the annihilating particles and given by

$$
v_{\mathrm{M} \varnothing \mathrm{l}}=2 \beta=2 \sqrt{1-\frac{4 m_{X}^{2}}{s}} .
$$

The rescaled cross section $\tilde{\sigma}$ defined above is dimensionless and function only of $x / z=$ $m_{X} / \sqrt{s}$ (or $\beta$ ) for the case of annihilation into massless gauge bosons and it always vanishes at threshold. Then it is easy to see that since we integrate in both $x, z$, the main dependence on the charged relic mass is contained in the prefactor in eq. (41) and can be reabsorbed in a rescaling of $Y_{X} \rightarrow Y_{X} / m_{X}$. For this reason we obtain nearly exactly $Y_{X} \propto m_{X}$ if there is no other mass scale involved. Note that in principle a much weaker logarithmic dependence on $m_{X}$ is present in the value of the freeze-out temperature, when $Y$ begins to deviate from $Y_{e q}$.

We are here computing the yield of the particle $X$ and to obtain the yield of particle and antiparticle we multiply by a factor of 2 or divide the cross section by $1 / 2$, since we are assuming $n_{X}=n_{\bar{X}}$. Also note that, contrary to intuition, for a particle with internal degrees of freedom like a coloured state, the total yield is the solution of the Boltzmann equation (4) with the cross section averaged over the initial states. Instead the yield per degree of freedom is obtained from the cross section averaged over $X$, but summed over

\footnotetext{
${ }^{2}$ Note that our definition differs from the one in [24] by a factor $m_{X}^{2} / x^{2}$ since we prefer to work with a dimensionless quantity and to absorb here all the dependence on $x$.
} 
$\bar{X}$ 3. The presence of many degrees of freedom in the initial state has then the effect of partially compensating the large cross section coming from the multiplicity of the final states.

\section{Annihilation cross section for a charged particle into gauge bosons}

\subsection{Abelian case}

For an abelian gauge symmetry, there are only three Feynman diagrams contributing to the annihilation cross section, analogous to those shown in Fig. 1; the t- and u-channel exchange of the scalar particle itself, and the 4-boson vertex. The amplitude is symmetric in the exchange of the gauge bosons and for a particle of charge $e_{X} g_{1}$ it is given by

$$
\mathcal{A}^{\mu \nu}=i g_{1}^{2} e_{X}^{2}\left[\frac{\left(2 p_{1}-p_{3}\right)^{\mu}\left(2 p_{2}-p_{4}\right)^{\nu}}{t-m_{X}^{2}}+\frac{\left(2 p_{1}-p_{4}\right)^{\nu}\left(2 p_{2}-p_{3}\right)^{\mu}}{u-m_{X}^{2}}+2 g^{\mu \nu}\right] \text {. }
$$

The cross section is a function of the mass and charge of the relic:

$$
\begin{aligned}
\sigma_{\mathrm{a} b}\left(m_{X}, s\right)= & \frac{4 \pi \alpha_{1}^{2} e_{X}^{4}}{s-4 m_{X}^{2}}\left[\sqrt{1-\frac{4 m_{X}^{2}}{s}}\left(1+\frac{4 m_{X}^{2}}{s}\right)\right. \\
& \left.+\frac{4 m_{X}^{2}}{s}\left(1-\frac{2 m_{X}^{2}}{s}\right) \log \left(\frac{1-\sqrt{1-\frac{4 m_{X}^{2}}{s}}}{1+\sqrt{1-\frac{4 m_{X}^{2}}{s}}}\right)\right]
\end{aligned}
$$

where $\alpha_{1}=g_{1}^{2} /(4 \pi)$ is the gauge coupling; note that a symmetry factor $1 / 2$ has to be added due to the symmetric final state of identical particles. For the rescaled cross section this gives

$$
\tilde{\sigma}_{\mathrm{a} b}(\beta)=8 \pi \alpha_{1}^{2} e_{X}^{4} \beta\left[1-\frac{1}{2} \beta^{2}+\frac{1-\beta^{4}}{4 \beta} \log \left(\frac{1-\beta}{1+\beta}\right)\right],
$$

which is a function only of $\beta=\sqrt{1-4 m_{X}^{2} / s}$ and the charge of the particle.

\subsection{Non-abelian case}

The computation for the annihilation into non-abelian gauge bosons is slightly more involved, since there is an additional contribution from the Feynman diagram with a gauge boson in the s-channel and the 3-gauge-boson vertex. The amplitude can be divided into a symmetric and an antisymmetric piece in the group indices. The symmetric one is analogous to the abelian case:

$$
\begin{aligned}
\mathcal{A}_{s y m}^{\mu \nu}= & i \frac{g_{N}^{2}}{2}\left\{T^{a}, T^{b}\right\}_{j i}\left[\frac{\left(2 p_{1}-p_{3}\right)^{\mu}\left(2 p_{2}-p_{4}\right)^{\nu}}{t-m_{X}^{2}}\right. \\
& \left.+\frac{\left(2 p_{1}-p_{4}\right)^{\nu}\left(2 p_{2}-p_{3}\right)^{\mu}}{u-m_{X}^{2}}+2 g^{\mu \nu}\right]
\end{aligned}
$$

\footnotetext{
${ }^{3}$ In fact any rescaling of the cross section by a factor $p$ due to a different counting of the degrees of freedom can be absorbed into a rescaling $1 / p$ of the yield(s).
} 

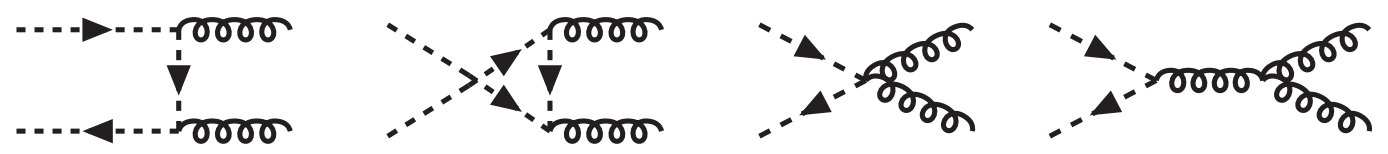

Figure 1: Feynman diagrams for the annihilation into gauge bosons, here for the case of gluons. In the abelian case, there is no 3-gauge-boson vertex, so the last diagram is absent.

while the antisymmetric part is given by

$$
\begin{aligned}
\mathcal{A}_{\text {asym }}^{\mu \nu}= & -i \frac{g_{N}^{2}}{2}\left[T^{a}, T^{b}\right]_{j i}\left[\frac{\left(2 p_{1}-p_{3}\right)^{\mu}\left(2 p_{2}-p_{4}\right)^{\nu}}{t-m_{X}^{2}}-\frac{\left(2 p_{1}-p_{4}\right)^{\nu}\left(2 p_{2}-p_{3}\right)^{\mu}}{u-m_{X}^{2}}\right. \\
& \left.+2 \frac{g^{\mu \nu}(t-u)-\left(2 p_{4}+p_{3}\right)^{\mu}\left(p_{1}-p_{2}\right)^{\nu}+\left(p_{1}-p_{2}\right)^{\mu}\left(2 p_{3}+p_{4}\right)^{\nu}}{s}\right] .
\end{aligned}
$$

The two contributions do not interfere due to the different symmetry, so we have for the amplitude squared, summing only over physical polarisations of the final gauge bosons:

$$
\begin{aligned}
|\mathcal{M}|^{2}= & 4 g_{N}^{4}\left\{\left|\left\{T^{a}, T^{b}\right\}_{j i}\right|^{2}\left[\frac{1}{2}+\frac{2 m_{X}^{4}}{\left(t-m_{X}^{2}\right)^{2}}+\frac{2 m_{X}^{2}}{t-m_{X}^{2}}\left(1-\frac{2 m_{X}^{2}}{s}\right)\right]\right. \\
& +\left|\left[T^{a}, T^{b}\right]_{j i}\right|^{2}\left[\frac{1}{2} \frac{\left(s+2\left(t-m_{X}^{2}\right)\right)^{2}}{s^{2}}+\frac{4 m_{X}^{2}}{s}+\frac{2 m_{X}^{4}}{\left(t-m_{X}^{2}\right)^{2}}\right. \\
& \left.\left.+\frac{2 m_{X}^{2}}{t-m_{X}^{2}}\left(1+\frac{2 m_{X}^{2}}{s}\right)\right]\right\} .
\end{aligned}
$$

Then the sum over all final and initial states for a scalar in the fundamental representation $T^{a}$ of the gauge group $S U(N)$, normalised such that $\operatorname{Tr}\left(T^{a} T^{b}\right)=\delta^{a b} / 2$, can be obtained from the usual group invariants:

$$
\begin{aligned}
\sum_{j, i, a, b} \frac{1}{2}\left|\left\{T^{a}, T^{b}\right\}_{j i}\right|^{2} & =\sum_{a, b} \frac{1}{2}\left(\frac{1}{N} \delta^{a b}+\frac{1}{2} \sum_{c}\left|d_{a b c}\right|^{2}\right) \\
& =C_{F}(N)\left(1+\frac{1}{2}\left(C_{A}^{2}(N)-4\right)\right)=\frac{\left(N^{2}-1\right)\left(N^{2}-2\right)}{4 N}
\end{aligned}
$$

where we have separated the singlet and adjoint contributions to the symmetric part for later convenience, included a factor $1 / 2$ for identical particles in the final states and used the Casimir invariants for the fundamental and adjoint representations, $C_{F}(N)=$ $\frac{N^{2}-1}{2 N}, C_{A}(N)=N$. Note that the ratio of the singlet to adjoint contributions is given simply by $\frac{2}{N^{2}-4}$. The antisymmetric channel instead gives

$$
\sum_{j, i, a, b} \frac{1}{2}\left|\left[T^{a}, T^{b}\right]_{j i}\right|^{2}=\frac{N^{2}-1}{4} C_{A}(N)=\frac{N\left(N^{2}-1\right)}{4} .
$$

Finally we obtain for the cross section averaged over initial states:

$$
\sigma_{n a b}\left(m_{X}, s\right)=\frac{\pi \alpha_{N}^{2}}{s-4 m_{X}^{2}} \frac{\left(N^{2}-1\right)^{2}}{N^{3}} \times
$$




$$
\begin{aligned}
& {\left[\sqrt{1-\frac{4 m_{X}^{2}}{s}}\left(1+\frac{4 m_{X}^{2}}{s}-\frac{N^{2}}{3\left(N^{2}-1\right)}\left(1-\frac{10 m_{X}^{2}}{s}\right)\right)\right.} \\
& \left.+4 \frac{m_{X}^{2}}{s}\left(1+\frac{2}{N^{2}-1} \frac{m_{X}^{2}}{s}\right) \log \left(\frac{1-\sqrt{1-\frac{4 m_{X}^{2}}{s}}}{1+\sqrt{1-\frac{4 m_{X}^{2}}{s}}}\right)\right] .
\end{aligned}
$$

This result coincides for $N=3$ with that reported in [26] for the Born cross section of a pair of gluons into squarks, allowing for the exchange of the initial and final state.

Then the rescaled cross section for $S U(N)$ is

$$
\begin{aligned}
\tilde{\sigma}_{n a b}(\beta)= & 2 \pi \alpha_{N}^{2} \frac{\left(N^{2}-1\right)^{2}}{N^{3}} \beta\left[1+\frac{N^{2}}{4\left(N^{2}-1\right)}-\frac{\beta^{2}}{2}\left(1+\frac{5 N^{2}}{6\left(N^{2}-1\right)}\right)\right. \\
& \left.+\frac{1-\beta^{2}}{2 \beta}\left(1+\frac{1}{2\left(N^{2}-1\right)}-\frac{\beta^{2}}{2\left(N^{2}-1\right)}\right) \log \left(\frac{1-\beta}{1+\beta}\right)\right] .
\end{aligned}
$$

Note that the contribution of order $\beta$ in the expression above in the limit $\beta \rightarrow 0$ is due to the symmetric part of the matrix element and that the antisymmetric piece instead vanishes at that order. Therefore the symmetric part of the cross section dominates at threshold.

So we see that for a non-abelian interaction the cross section is larger than for the abelian case, not only due to the possibly larger coupling $\alpha_{N}$, but also due to the opening of an antisymmetric channel and of course to the multiplicity of the final states. In fact for large $N$ the averaged cross section increases as $N$ and therefore the yield decreases as $1 / N$.

\subsection{Annihilation into $S U(N)$ gauge boson and photon}

The annihilation cross section into gluon and photon is just the same as the abelian one, but with a different vertex for the gluon. Then considering a particle of electromagnetic charge $e_{X} g_{1}$, in the representation $T^{a}$ of the gauge group $S U(N)$ with coupling $g_{N}$, annihilating with its own antiparticle, the amplitude is given by 4

$$
\mathcal{A}^{\mu \nu}=i g_{1} e_{X} g_{N} T_{j i}^{a}\left[\frac{\left(2 p_{1}-p_{3}\right)^{\mu}\left(2 p_{2}-p_{4}\right)^{\nu}}{t-m_{X}^{2}}+\frac{\left(2 p_{1}-p_{4}\right)^{\nu}\left(2 p_{2}-p_{3}\right)^{\mu}}{u-m_{X}^{2}}+2 g^{\mu \nu}\right] \text {. }
$$

From this we easily obtain the cross section as:

$$
\begin{aligned}
\sigma_{1 N}\left(m_{X}, s\right)= & \frac{8 \pi \alpha_{1} \alpha_{N} e_{X}^{2}}{s-4 m_{X}^{2}}\left|T_{j i}^{a}\right|^{2}\left[\sqrt{1-\frac{4 m_{X}^{2}}{s}}\left(1+\frac{4 m_{X}^{2}}{s}\right)\right. \\
& \left.+\frac{4 m_{X}^{2}}{s}\left(1-\frac{2 m_{X}^{2}}{s}\right) \log \left(\frac{1-\sqrt{1-\frac{4 m_{X}^{2}}{s}}}{1+\sqrt{1-\frac{4 m_{X}^{2}}{s}}}\right)\right],
\end{aligned}
$$

\footnotetext{
${ }^{4}$ Strictly speaking, in this case the final state particles are different and therefore there are no independent $\mathrm{t}$ - and $\mathrm{u}$-channels, but we can still write the amplitude to be symmetric in $\mathrm{t}$ and $\mathrm{u}$ in order to make direct contact with the previous results.
} 
where $\alpha_{1, N}$ are the gauge couplings and the symmetry factor $1 / 2$ in this case is absent since the final particles are not identical.

Averaging over the initial and summing over the final state, we have

$$
\frac{1}{N} \sum_{j, i, a} T_{j i}^{a} T_{i j}^{a}=\frac{1}{N} \sum_{j, i} C_{F}(N) \delta_{i j}=\frac{N^{2}-1}{2 N}
$$

for the fundamental representation. This gives for the rescaled cross section

$$
\tilde{\sigma}_{1 N}(\beta)=8 \pi \alpha_{1} \alpha_{N} e_{X}^{2} \frac{N^{2}-1}{N} \beta\left[1-\frac{1}{2} \beta^{2}+\frac{1-\beta^{4}}{4 \beta} \log \left(\frac{1-\beta}{1+\beta}\right)\right]
$$

which is a factor $\left(N^{2}-1\right) \alpha_{N} /\left(N \alpha_{1} e_{X}^{2}\right)$ larger than the pure $U(1)$ contribution. Again the cross section increases as $N$ for large $N$.

\subsection{Annihilation into physical $\mathrm{Z}$ and $S U(N)$ gauge boson/photon}

The annihilation cross section into massive $\mathrm{Z}$ and photon/SU(N) gauge boson has the same form as the abelian one. We consider here a particle with Z-coupling $g_{1} e_{Z}$, in the representation $T^{a}$ of the gauge group $S U(N)$ with coupling $g_{N}$, annihilating with its own antiparticle and we obtain

$$
\mathcal{A}^{\mu \nu}=i g_{1} e_{Z} g_{N} T_{j i}^{a}\left[\frac{\left(2 p_{1}-p_{3}\right)^{\mu}\left(2 p_{2}-p_{4}\right)^{\nu}}{t-m_{X}^{2}}+\frac{\left(2 p_{1}-p_{4}\right)^{\nu}\left(2 p_{2}-p_{3}\right)^{\mu}}{u-m_{X}^{2}}+2 g^{\mu \nu}\right]
$$

where $p_{4}$ is the $\mathrm{Z}$ boson momentum obeying $p_{4}^{2}=M_{Z}^{2}$; the annihilation into photon and $\mathrm{Z}$ is easily read off by taking just $g_{N} T_{j i}^{a} \rightarrow g_{1}^{\prime} e_{X}$. Then we easily obtain the cross section as:

$$
\begin{aligned}
\sigma_{\mathrm{ZN}}\left(m_{X}, M_{Z}, s\right)= & \frac{8 \pi \alpha_{1} \alpha_{N} e_{Z}^{2}}{s-4 m_{X}^{2}}\left|T_{j i}^{a}\right|^{2}\left[\sqrt{1-\frac{4 m_{X}^{2}}{s}}\left(1-\frac{M_{Z}^{2}}{s}+\frac{4\left(m_{X}^{2}-M_{Z}^{2}\right)}{s-M_{Z}^{2}}\right)\right. \\
& \left.+\frac{4 m_{X}^{2}}{s}\left(1-\frac{5 M_{Z}^{2}}{8 m_{X}^{2}}-\frac{4 m_{X}^{2}-3 M_{Z}^{2}}{2\left(s-M_{Z}^{2}\right)}\right) \log \left(\frac{1-\sqrt{1-\frac{4 m_{X}^{2}}{s}}}{1+\sqrt{1-\frac{4 m_{X}^{2}}{s}}}\right)\right]
\end{aligned}
$$

where $\alpha_{1, N}$ are the gauge couplings.

Averaging over the initial and summing over the final state as in eq. (20), we have for the rescaled cross section

$$
\begin{aligned}
\tilde{\sigma}_{1 N}\left(\beta, a_{Z}\right)= & 8 \pi \alpha_{1} \alpha_{N} e_{Z}^{2} \frac{N^{2}-1}{2 N} \beta\left[1-a_{Z}\left(1-\beta^{2}\right)+\frac{\left(1-4 a_{Z}\right)\left(1-\beta^{2}\right)}{1-a_{Z}\left(1-\beta^{2}\right)}\right. \\
& \left.+\frac{1-\beta^{2}}{\beta}\left(1-\frac{5}{2} a_{Z}-\left(1-\beta^{2}\right) \frac{1-3 a_{Z}}{2-2 a_{Z}\left(1-\beta^{2}\right)}\right) \log \left(\frac{1-\beta}{1+\beta}\right)\right],
\end{aligned}
$$

where $a_{Z}=M_{Z}^{2} / m_{X}^{2}$. Note that the cross section for annihilation into photon and Z, is given by the substitution $\alpha_{N} \frac{N^{2}-1}{2 N} \rightarrow \alpha_{1}^{\prime} e_{X}^{2}$. For the specific case of the right-handed stau (stop), the coupling with the $\mathrm{Z}$ boson and photon are respectively given by $e_{Z}^{2} \alpha_{1}=$ $\alpha_{e m} \tan ^{2} \theta_{W}\left(e_{Z}^{2} \alpha_{1}=4 / 9 \alpha_{e m} \tan ^{2} \theta_{W}\right)$ and $e_{X}^{2} \alpha_{1}^{\prime}=\alpha_{e m}\left(e_{X}^{2} \alpha_{1}^{\prime}=4 / 9 \alpha_{e m}\right)$, where $\theta_{W}$ is the Weinberg angle. 


\subsection{Annihilation into massless EW gauge bosons}

The cross section for annihilation into massless $S U(2)_{L}$ gauge bosons can be obtained directly from the general formula for the non-abelian case. One has to take into account, however, that in this case the scalar $S U(2)_{L}$ doublet is not degenerate in mass and that the initial particles can be a mixture of left- and right-chiral states. We neglect here the effects of EW symmetry breaking; the results are hence applicable for the case of a heavy relic that decouples before EW symmetry breaking takes place.

Considering the scalar relic to be $X=X_{L} \cos \theta+X_{R} \sin \theta$ and denoting with $m_{X^{\prime}}$ the mass of its left-handed doublet partner, which is sufficiently larger than $m_{X}$ to neglect coannihilations, we obtain for the annihilation cross section into $W^{1,2}$ gauge bosons:

$$
\begin{aligned}
& \sigma_{W 2}\left(s, m_{X}, m_{X^{\prime}}\right)= \\
& \frac{2 \pi \alpha_{2}^{2} \cos ^{4} \theta}{s-4 m_{X}^{2}}\left[\sqrt{1-\frac{4 m_{X}^{2}}{s}}\left(\frac{2}{3}+\frac{13}{3} \frac{m_{X}^{2}}{s}-\frac{m_{X^{\prime}}^{2}}{s}+\frac{\left(m_{X}^{2}+m_{X^{\prime}}^{2}\right)^{2}}{s m_{X^{\prime}}^{2}+\left(m_{X^{\prime}}^{2}-m_{X}^{2}\right)^{2}}\right)\right. \\
& \left.\quad+2\left(\frac{m_{X^{\prime}}^{2}+m_{X}^{2}}{s}-\frac{\left(m_{X^{\prime}}^{2}-m_{X}^{2}\right)^{2}}{2 s^{2}}\right) \log \left(\frac{s+2\left(m_{X^{\prime}}^{2}-m_{X}^{2}\right)-\sqrt{s\left(s-4 m_{X}^{2}\right)}}{s+2\left(m_{X^{\prime}}^{2}-m_{X}^{2}\right)+\sqrt{s\left(s-4 m_{X}^{2}\right)}}\right)\right],
\end{aligned}
$$

while the annihilation into $W^{3}$ is similar to the abelian one in eq. (9) for $e_{X}=\cos \theta / 2$. Note that the cross section is suppressed by the mixing angle as $\cos ^{4} \theta$ and by the fact that the group indices are not summed for the initial state. Also in this case the rescaled cross section is not just a simple function of $\beta$, but also of the mass difference in the doublet. We have in fact

$$
\begin{gathered}
\tilde{\sigma}_{W 2}\left(\beta, \delta^{2}\right)=2 \pi \alpha_{2}^{2} \cos ^{4} \theta \beta\left[\frac{5}{2}+\frac{11}{6} \beta^{2}-\delta^{2}+\frac{4 \beta^{2} \delta^{4}}{\left(1+2 \delta^{2}\right)^{2}-\beta^{2}}\right. \\
\left.+\frac{1-\beta^{2}+2 \delta^{2}-\delta^{4}}{\beta} \log \left(\frac{1+2 \delta^{2}-\beta}{1+2 \delta^{2}+\beta}\right)\right],
\end{gathered}
$$

where $\delta^{2}=\left(m_{X^{\prime}}^{2}-m_{X}^{2}\right) / s$. The cross section still vanishes for $\beta=0$ and is finite for $\delta^{2} \rightarrow \infty$. The detailed expressions for the case of broken EW symmetry are much more involved and include also the contribution of the Higgs s-channel allowing for resonance enhancement. They are given in Appendix B.

\subsection{Sommerfeld enhancement}

In the previous sections we have computed the annihilation cross sections to lowest order in the gauge coupling. However, it was shown long ago [17] that an expansion in terms of the coupling is inadequate close to threshold, where the velocities of the annihilating particles go to zero,

$$
\beta \equiv \sqrt{1-\frac{4 m_{X}^{2}}{s}} \rightarrow 0 .
$$

The enhancement at low velocities becomes apparent when one computes the one-loop corrections, which are enhanced by a factor $\frac{C \alpha \pi}{2 \beta}$. Here, $C$ is a process-dependent constant, $\alpha$ is the gauge coupling of the annihilating scalars, $\alpha_{1}$ in the case of $U(1)$ boson exchanges, or $\alpha_{N}$ for $S U(N)$ gauge boson exchanges, respectively. To account for this long-distance 
effect, one therefore has to resum a whole class of diagrams, which consist of $t$-channel ladder-type exchanges of massless soft Coulomb $S U(N)$ or $U(1)$ gauge bosons between the annihilating charged particles.

This resummation of terms $\sim \alpha^{n} / \beta^{n}$ leads to the so-called Sommerfeld factor which multiplies the lowest-order annihilation cross section. The Sommerfeld enhancement is given by the modulus squared of the particle wave function at the origin,

$$
E \equiv|\Psi(0)|^{2}=\frac{z}{1-\exp (-z)}, \quad z=\frac{C \alpha \pi}{\beta} .
$$

Because this effect is a long-distance one, taking place at a scale $\sim \beta m_{X}$, it factorises from the annihilation cross section which is a short-distance effect at the hard-scattering scale of order of the mass $m_{X}$. Schematically,

$$
\sigma^{\mathrm{SF}}\left(\beta, m_{X}\right)=E\left(\alpha\left(\beta m_{X}\right)\right) \times \sigma^{0}(\beta) .
$$

Here, $\sigma^{0}$ is the leading-order annihilation cross section, which has been presented in the preceding subsections. Eq. (29) is in principle only valid if the annihilating partons are in a single $S U(N)$ channel, i.e. for particle in the fundamental representation either in the singlet or adjoint configurations. If multiple channels $c$ contribute, eq. (29) has to be modified to

$$
\sigma^{\mathrm{SF}}\left(\beta, m_{X}\right)=\sum_{c} E_{c}\left(\alpha\left(\beta m_{X}\right)\right) \times \sigma_{c}^{0}(\beta) .
$$

Here, $\sigma_{c}^{0}(\beta)$ is the projection of the leading-order annihilation cross section in the relevant channel. For a scalar in the fundamental representation of the $S U(N)$ gauge group annihilating into massless $S U(N)$ gauge bosons, we have seen that only the contribution proportional to the group-symmetric part survives in the limit of vanishing $\beta$ and is enhanced at low velocities. Therefore at leading order the cross sections $\sigma_{c}^{0}$ can be taken to be the same for the singlet and adjoint part up to colour factors and proportional to the total cross section given in eq. (17) 5. We note also that due to the presence of more than one channel, the Sommerfeld factor for an $S U(N)$ gauge theory becomes dependent also on the final states, since not all channels may contribute to the annihilation into a given final state.

However, the presence of the thermal bath complicates things, as the interactions with the background gauge bosons may prevent the annihilating partons to be initially in a definite $S U(N)$ channel. The time scales for the Sommerfeld effect and the interactions with the thermal bath are of competing order, so it is not clear how strong such effect can be. In this paper we will consider both extreme situations, i.e. the case when the thermal bath has no effect and the case when there is no definite initial channel. In the latter case, it was argued in the literature that due to the mixing of states one should just take an average $C^{a v}$ extracted from the averaged one-loop correction, leading again to a single Sommerfeld factor as in eq. (29) (see, for example, ref. [27]). While the two approaches give identical results by construction at first order, they correspond to two quite distinct resummations of the higher orders and they are numerically substantially different.

We obtained the coefficients $C$ by computing the $1 / \beta$-enhanced contributions for $t$ channel $S U(N)$ gauge boson exchange at one loop in the threshold expansion (see for

\footnotetext{
${ }^{5}$ Taking the true $\sigma_{\mathbf{1}}^{0}$ and $\sigma_{\mathbf{A}}^{0}$ instead, differs from the total $\sigma^{0}$ only in the terms suppressed by $\beta^{2}$ and amounts to a correction smaller than $1 \%$ at threshold where the Sommerfeld factor is effective.
} 
example [28] and references therein). For the generation of the relevant one-loop graphs and the Lorentz algebra we used the Mathematica packages FeynArts and FeynCalc [29]. We simplified the resulting expressions to only keep terms that are leading in $\beta$, that is, we only kept terms that are enhanced in the soft region of the one-loop integrals, which were then simple enough to perform by hand. Alternatively, as mentioned above, one obtains the form (28) directly by computing the normalised wave function at the origin from the Schrödinger equation, describing the annihilating parton pair, with a Coulomb interaction potential for positive energies $\sim \beta^{2} m_{X}$ [17].

The Sommerfeld enhancement due to exchanges of massless Coulomb $S U(N)$ gauge bosons is the same for the singlet channel of annihilation into $S U(N)$ gauge bosons $B_{N}$ and the annihilation into $U(1)$ gauge bosons $B_{1}$,

$$
C_{S \bar{S} \rightarrow B_{N} B_{N}}^{1}=C_{S \bar{S} \rightarrow B_{1} B_{1}}=C_{F}(N)=\frac{N^{2}-1}{2 N} .
$$

The factor for the adjoint channel is instead found to be negative and thus suppressing,

$$
C_{S \bar{S} \rightarrow B_{N} B_{N}}^{\mathbf{A}}=C_{F}(N)-\frac{C_{A}(N)}{2}=-\frac{1}{2 N} .
$$

The same factors $C^{\mathbf{1}}$ or $C^{\mathbf{A}}$ apply also for other final states of the singlet or adjoint channels. For example, the Sommerfeld factor for $\tilde{t}^{*} \rightarrow h h$ is $C_{S U(3)}^{1}=4 / 3$, while that for $\tilde{t t^{*}} \rightarrow g h, g \gamma, g Z$ is $C_{S U(3)}^{\mathbf{A}}=-1 / 6$.

Even if the adjoint channel leads to a suppression, upon summing over both contributions in eq. (30), the net effect is still quite enhancing for small $N$. We have then in fact

$$
\sigma^{\mathrm{SFsum}}\left(\beta, m_{X}\right)=\sigma^{0}(\beta)\left[E_{\mathbf{1}}\left(\alpha\left(\beta m_{X}\right)\right) \times \frac{2}{N^{2}-2}+E_{\mathbf{A}}\left(\alpha\left(\beta m_{X}\right)\right) \times \frac{N^{2}-4}{N^{2}-2}\right],
$$

where, as described above, we have taken $\left(N^{2}-2\right) / 2 \sigma_{1}^{0}=\left(N^{2}-2\right) /\left(N^{2}-4\right) \sigma_{\mathbf{A}}^{0}=\sigma^{0}(\beta)$, and $\sigma^{0}(\beta)$ is given in eq. (17). For $S U(3)$ this gives

$$
\sigma_{S U(3)}^{\mathrm{SFsum}}\left(\beta, m_{X}\right)=\sigma_{S U(3)}^{0}(\beta) \frac{\pi \alpha_{3}}{42 \beta}\left[\frac{16}{1-e^{-\frac{4}{3} \frac{\pi \alpha_{3}}{\beta}}}-\frac{5}{1-e^{\frac{1}{6} \frac{\pi \alpha_{3}}{\beta}}}\right],
$$

so that the enhancement in the singlet dominates over the suppression in the adjoint channel.

On the other hand, averaging the one loop contribution over initial channels 6 results in a factor

$$
C_{S \bar{S} \rightarrow B_{N} B_{N}}=\frac{N^{2}+2}{2 N\left(N^{2}-2\right)}=: C_{S U(N)}^{\mathrm{av}},
$$

which is although enhancing, much less so than the net effect of the summation over singlet and adjoint channels. For $S U(3)$, this factor is $C_{S U(3)}^{\text {av }}=11 / 42$, leading to

$$
\sigma_{S U(3)}^{\mathrm{SFav}}\left(\beta, m_{X}\right)=\sigma_{S U(3)}^{0}(\beta) \frac{\pi \alpha_{3}}{42 \beta} \frac{11}{1-e^{-\frac{11 \pi \alpha_{3}}{42 \beta}}} .
$$

\footnotetext{
${ }^{6}$ Averaging over initial channels is not to be confused with averaging over initial states which is to be done in addition when solving the Boltzmann equation.
} 


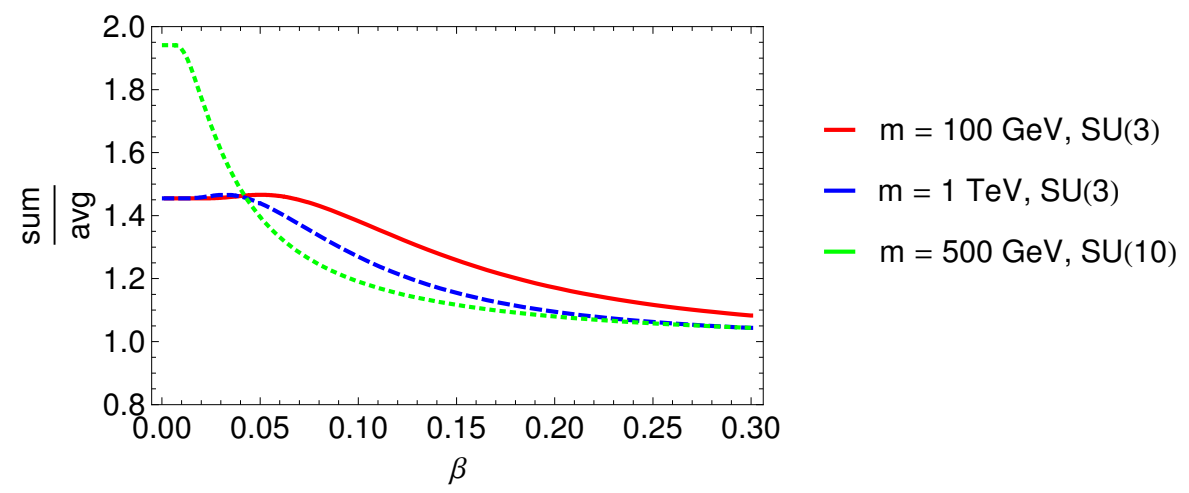

Figure 2: Ratio of summed over averaged Sommerfeld enhancement, $\sigma_{S U(3)}^{\mathrm{SF} s u m} / \sigma_{S U(3)}^{\mathrm{SFav}}$, as a function of $\beta$. The full red line shows the $S U(3)$ case for a mass $m=100 \mathrm{GeV}$ and the dashed blue line for a mass $m=1 \mathrm{TeV}$; the dotted green line is for the hypothetical case of $S U(10)$ with $m=500 \mathrm{GeV}$.

Note that the first term of the expansion of eq. (36) coincides with the 1-loop result of [26] for $g g \rightarrow \tilde{q} \tilde{q}^{*}$ near threshold.

If the difference in the exponents in the denominators of eqs. (34) and (36) could be neglected the two expression would be equal. However, in the small $\beta$ region where the Sommerfeld enhancement is relevant, the difference amounts to up to $50 \%$ for $S U(3)$, and is even larger for hypothetical larger $N$, see Figure 2 ,

For scalars charged under a $U(1)$ group, there is of course a corresponding enhancement due to $U(1)$ boson exchanges. However, the enhancement factor is now governed by the $U(1)$ coupling, and thus weaker than an enhancement under a strong $S U(N)$ gauge group. The Sommerfeld factor for the dominant annihilation channel into $U(1)$ gauge boson pairs can very simply be determined from the abelian part of the calculation that led to the factor quoted above. We find,

$$
C_{S \bar{S} \rightarrow B_{1} B_{1}}=1
$$

for $t$-channel $U(1)$ exchange, and the coupling in eq. (28) is the $U(1)$ coupling $\alpha_{1}$.

Another issue regarding the thermal bath is the fact that gauge bosons acquire a mass through interactions with the plasma. This Debye screening effect happens at a scale of order $\sim g T$, whereas the Sommerfeld effect is of order $\sim \alpha m_{X} \beta \sim \alpha \sqrt{m_{X} T} \gg g T$. Thus the thermal masses of initially massless gauge bosons do not affect the Sommerfeld enhancement.

Finally, there are also massive gauge bosons such as $W \mathrm{~s}$ and $Z \mathrm{~s}$ to consider. The Sommerfeld factor arises from instantaneous Coulomb exchanges of massless gauge bosons between the slow moving annihilating pair close to threshold, thus resulting in an $1 / \beta$ enhancement, signalling the inadequacy of trying to describe this exchange in an expansion in terms of loop corrections. Naturally, massive gauge bosons have a finite width, and thus cannot be exchanged instantaneously. In terms of Feynman graphs, the momentum flowing through a massive gauge boson that is exchanged between the annihilating pair is naturally cut off by the mass of the exchanged boson and can never become too soft. The Sommerfeld effect is exponentially suppressed with the mass of the gauge boson, as an analysis of the wavefunction picture reveals. It can nevertheless become important for relics with masses much larger than the electroweak scale, as a very heavy Wino discussed 
in [19]. In the following we will consider only the case of massless gauge bosons, which is the dominant effect for coloured relics and for purely right-handed sleptons. For a more detailed discussion in case of massive EW gauge bosons we refer the reader to [19, 16].

\subsection{Unitarity bound}

We next compare the above cross sections with the unitarity bound. Using unitarity and partial wave expansion, the non-elastic cross section for a particle with spin $s_{p}$ is given by [15]

$$
\sigma_{n o n-e l, J}=\frac{4 \pi(2 J+1)\left(1-\eta_{J}^{2}\right)}{\left(2 s_{p}+1\right)^{2} \vec{p}_{i}^{2}}
$$

where $J$ is the angular momentum of the process, $\vec{p}_{i}$ is the initial particle momentum, $4 \vec{p}_{i}^{2}=s \beta^{2}$ in the centre of mass frame in our case, and $\eta_{J}^{2}$ is the contribution of the elastic part. This gives an upper bound for the annihilation cross section with angular momentum $J$ as

$$
\sigma_{a n n, J} \leq \frac{16 \pi(2 J+1)}{\left(2 s_{p}+1\right)^{2} s \beta^{2}} .
$$

The lowest value is obtained taking $J=0$ and since the s-wave annihilation is usually the dominant contribution for a scalar non-relativistic particle with $s_{p}=0$, we will take it as a reference value. We therefore have for the maximal rescaled cross section:

$$
\tilde{\sigma}_{\max }=16 \pi
$$

independent of the particle mass or energy. In this case the thermal averaging is simple and we obtain

$$
\left\langle\sigma_{\max } v\right\rangle_{x}=\frac{16 \pi}{x^{2}} \frac{K_{2}(2 x)}{K_{2}(x)^{2}},
$$

which we will consider in the following to be the maximal cross section per degree of freedom 7 . We see clearly that the cross sections discussed above satisfy this bound and are suppressed at the very least by $\alpha^{2}$. Figure 3 shows the rescaled cross sections for the abelian and non-abelian cases, eqs. (10) and (17), together with the unitarity bound eq. (40) as a function of the relative velocity of the annihilating particles.

The unitarity cross section $\tilde{\sigma}_{\max }$ can be used to obtain a lower bound of the yield. Moreover, it can be taken as the maximal annihilation cross section possible even after the QCD phase transition, when the coloured states are confined into the equivalent of scalar hadrons and fermionic mesons [30]. Constraints from cosmology on such kind of hadronic states have been mostly studied for the case of a stable exotic quark [14, a gluino LSP [27] or for very long-lived gluino in the split SUSY scenarios [18. It has been argued in 31 that the annihilation cross section for such states could become much stronger, if bound states between two scalar hadrons/fermionic mesons are formed with rate $\sim \pi / \Lambda_{Q C D}^{2}$ and in that case the coloured relic abundance after the QCD phase transition is further reduced below $Y \sim 10^{-16}-10^{-17}$. We will not consider this possibility in the following, but note however that, while most of the cosmological bounds for a decaying relicare then satisfied, one still needs to consider the bounds for a stable relic.

\footnotetext{
${ }^{7}$ Note that here we are computing explicitly in the centre of mass frame, while the Boltzmann equation requires to use the covariant or lab frame. The difference between the two frames has been discussed in 24] and gives only a small correction for non-relativistic particles, which we neglect here.
} 


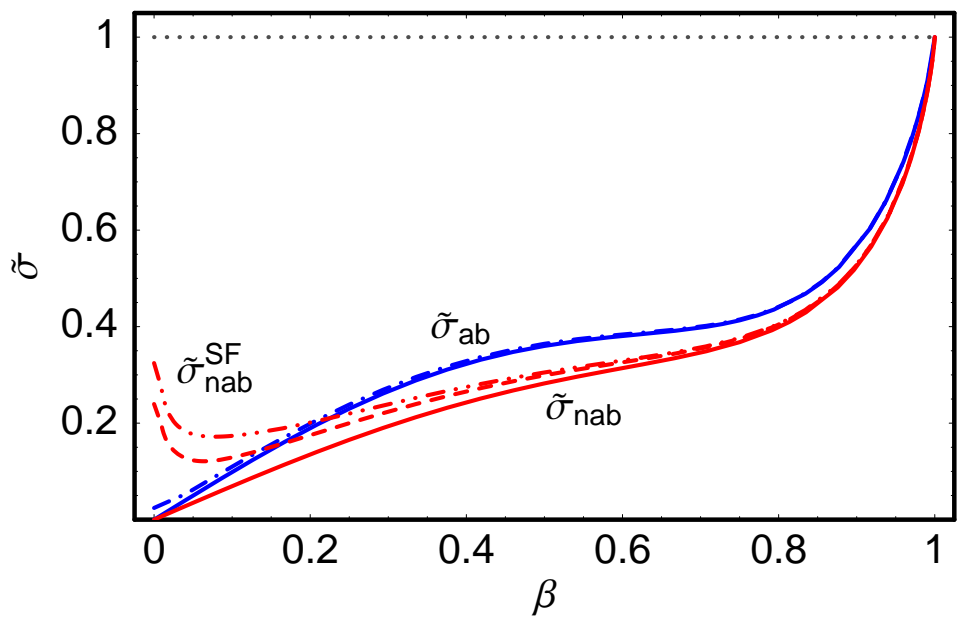

Figure 3: Dependence of the rescaled cross sections on the relative velocity $\beta$, normalised to 1 at large $s$, i.e. $\beta=1$. The solid lines show the leading order section, the dashed/dashed dotted lines the effect of the Sommerfeld enhancement, that makes the cross sections non-vanishing at the threshold $\beta=0$. The $S U(3)$ cross sections are the upper (red) lines, including the averaged Sommerfeld factor in the dashed line and the summed one in the dash-dotted. For the abelian case (blue lines) the Sommerfeld effect is much milder and shown in the dash-dotted line. Note that the region for $\beta \sim 0$ contributes more strongly to the thermally averaged cross section due to the Boltzmann-suppression for large $\beta$.

\subsection{Thermally averaged cross sections and velocity expansion}

We integrate eq. (5) numerically to obtain the thermally averaged cross section. Very often such a quantity is instead approximated with the first terms of its velocity expansion, since the relevant regime takes place when the annihilating particles are already non-relativistic. To obtain such an expansion, one can use the approximation

$$
s-4 m_{X}^{2} \simeq 4 m_{X}^{2} \beta^{2}
$$

and expand in $\beta$ the expression

$$
\sigma v_{\mathrm{M} \varnothing \mathrm{l}} \simeq \frac{1}{2 m_{X}^{2} \beta} \tilde{\sigma}(\beta) .
$$

We see that if $\tilde{\sigma}$ is constant at zero velocity, the cross section is enhanced like $1 / \beta$ in that limit. This is indeed the case both for the Sommerfeld-enhanced cross section and the unitarity one.

The first term in the expansion, which is independent of the velocity and coincides therefore with the first term in the expansion of the thermally averaged cross section [24], is given by

$$
\begin{aligned}
\sigma_{a b} v & \rightarrow \frac{2 \pi \alpha_{1}^{2} e_{X}^{4}}{m_{X}^{2}}+\mathcal{O}\left(\beta^{2}\right), \\
\sigma_{n a b} v & \rightarrow \frac{\pi \alpha_{N}^{2}}{m_{X}^{2}} \frac{\left(N^{2}-1\right)\left(N^{2}-2\right)}{4 N^{3}}+\mathcal{O}\left(\beta^{2}\right),
\end{aligned}
$$




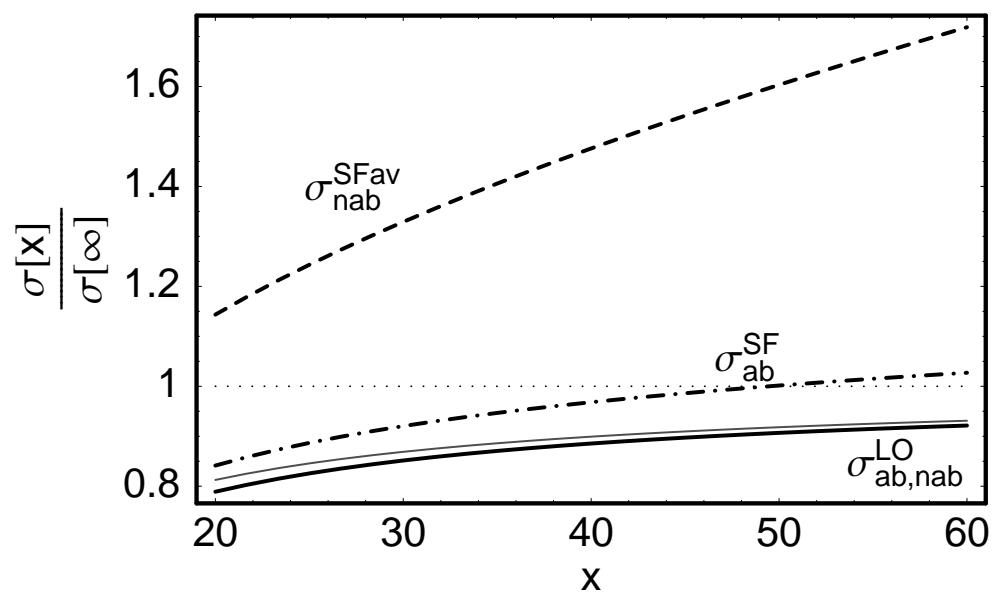

Figure 4: Ratio of the thermally-averaged cross section and the first term in the velocity expansion around $\beta=0$, for $m_{X}=350 \mathrm{GeV}$. The thick solid line is for the abelian, the thin line for the non-abelian $(S U(3))$ case. Dash-dotted and dashed respectively are the same ratios including the Sommerfeld enhancement, only the averaged one for the non-abelian case: we see that in this case the thermally averaged cross sections do not converge to the first order term in velocity, but that the latter can still give a good estimate within $15 \%$ of the full result in the abelian case; for the non-abelian case the Sommerfeld enhancement changes the result considerably and the velocity expansion fails. Note that the case of the summed Sommerfeld factor is outside the range of the plot.

for the abelian and non-abelian cases respectively.

We plot in Figure 4 the thermally averaged cross sections as a function of $x$ normalised with respect to the first term in their velocity expansion including also the Sommerfeld enhancement factor, both for the abelian case and for the QCD case with $N=3$. We see that keeping only the lowest order overestimates the thermally averaged cross section, i.e. underestimates the yield, in the abelian case by at most $20 \%$ in the region of freezeout $(x \sim 30)$. The non-abelian case for $N=3$ is approximated better also because the freeze-out takes place at a larger $x \sim 40$, i.e. smaller $\beta$. On the other hand, once we include the Sommerfeld enhancement, the thermally averaged cross section does no more converge to the first constant term in the velocity expansion due to the threshold singularity at $\beta=0$. Nevertheless the first order term without the enhancement can still give a reasonably good approximation for the abelian case, since the Sommerfeld enhancement partially compensate the $20 \%$ underestimation of the Born result. For the non-abelian case the Sommerfeld enhancement is so strong that the low energy expansion can give only an order of magnitude estimate.

\section{Results for the relic density}

We solve the Boltzmann equation (4) numerically for the exact thermally averaged cross sections given above. This improves the old results [13] that were obtained with the velocity expansion.

For the case of an abelian charged relic, we consider $e_{X}= \pm 1$ and we set the coupling to be $\alpha_{e m}=1 / 128$. For the non-abelian case we take $N=3$ and $\alpha_{N}$ to be the QCD 


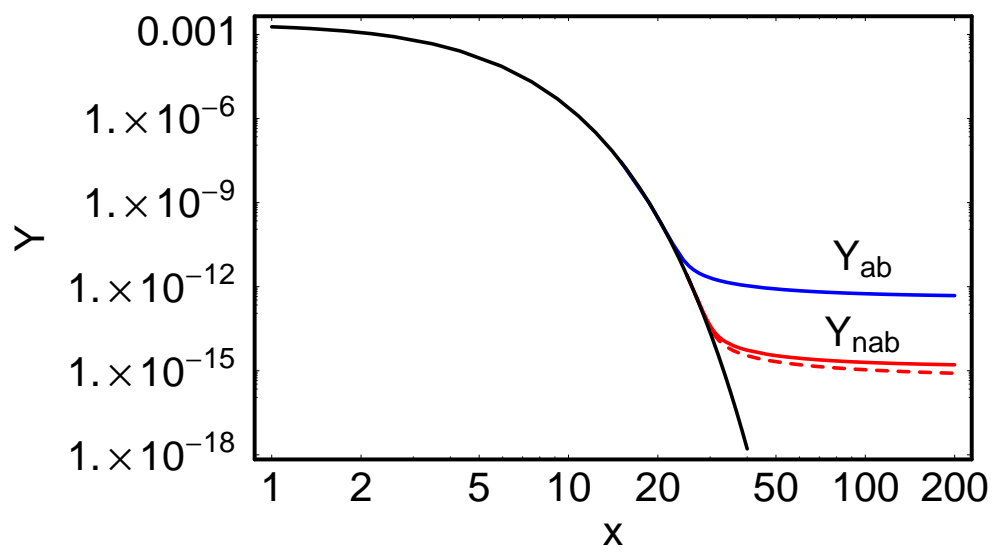

Figure 5: Time evolution of the particle yield for the cases of abelian and non-abelian cross section, for $m_{X}=200 \mathrm{GeV}$. The upper (blue) curve is for an electromagnetically charged scalar particle with unit charge, while the lower (red) curves correspond to a single coloured scalar in the fundamental representation without the Sommerfeld factor (solid) and with the Sommerfeld factor averaged (dashed). We see that the treatment of the Sommerfeld factor has an impact of about $30 \%$ on the final number density.

coupling $\alpha_{3}(Q)$ with $Q=2 m_{X}$ in the hard process and $Q=\beta m_{X}$ in the Sommerfeld correction, c.f. Sect. 3.6. In order to avoid the non-perturbative regime, we cut off the running of $\alpha_{3}$ at $Q=2 \mathrm{GeV}$, i.e. $\alpha_{3}(Q<2 \mathrm{GeV}) \equiv \alpha_{3}(2 \mathrm{GeV})$.

For the entropy and energy density parameters we take $g_{S}^{1 / 2}=g_{\rho}^{1 / 2}=10$, since we expect the freeze-out to take place between $10-100 \mathrm{GeV}$, when only the light Standard Model particles are still in equilibrium in the thermal bath.

Our results are plotted in Figure 5. We see that the yield $Y$ follows relatively closely the equilibrium density until the time of freeze-out, which happens at different values of $x$ for the different cross sections. As expected the non-abelian interactions being stronger gives a considerably lower relic density. The ratio between the two cases is well approximated by the ratio of cross sections, $\sigma_{n a b} v / \sigma_{a b} v$, at zero velocity :

$$
\frac{Y_{a b}}{Y_{n a b}}=\frac{7}{27} \frac{\alpha_{3}^{2}}{\alpha_{e m}^{2}} \approx 40 .
$$

We next consider the dependence on the only dimensional parameter, the mass of the charged relic. We have seen that the thermal average can be written only as a function of $x$ and since we are integrating the Boltzmann equation to $x \rightarrow \infty$ we get rid of the dependence on $m_{X}$ that is contained there. A subleading dependence would survive by integrating to a finite value of $x$, but this effect is negligible for the present universe with a temperature $T_{\text {now }} \sim 10^{-4} \mathrm{eV} \ll m_{X}$. On the other hand, the mass directly enters in the coefficient of eq. (4) and that is the stronger dependence on $m_{X}$. Note that this dependence is present even in the unitarity case, where the reduced cross section is explicitly independent of the mass and velocity. In general therefore the yield is proportional to the mass and can be rescaled as

$$
Y\left(m_{X}\right)=Y(1 \mathrm{TeV})\left(\frac{m_{X}}{1 \mathrm{TeV}}\right) .
$$




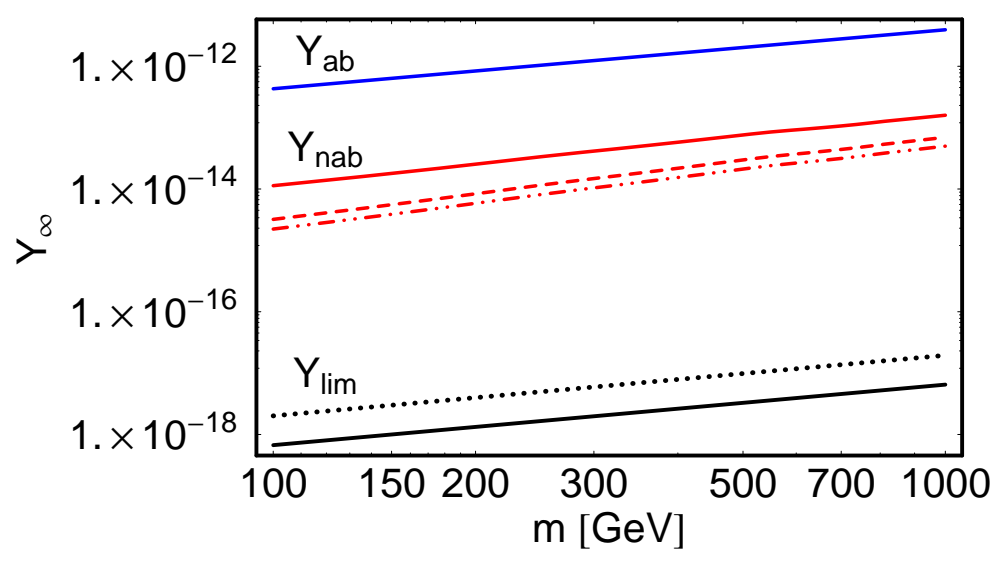

Figure 6: Dependence of the yield on the mass of the charged relic. From top to bottom, the first (blue) line is for the case of an electromagnetically charged relic, while the second (red) line is for a coloured relic, the dashed and dash-dotted lines include the Sommerfeld factor, averaged and summed respectively. The lower two (black) lines correspond to the maximal annihilation cross section given by unitarity - the solid one for a single d.o.f., the dotted one for 3 d.o.f. for the fundamental representation of QCD. Note that the non-abelian case is still three orders of magnitudes away from the unitarity cross section.

with $Y_{a b}(1 \mathrm{TeV})=3.9 \times 10^{-12}$ and $Y_{n a b}(1 \mathrm{TeV})=1.6 \times 10^{-13}$ for the abelian and nonabelian cases, respectively, for the total degrees of freedom, including antiparticles. For the case of the unitarity cross section, the total yield becomes instead $Y_{\text {lim }}(1 \mathrm{TeV})=6.6 \times 10^{-18}$ (or $2 \times 10^{-17}$ for three degrees of freedom).

Since the energy density also increases for larger masses, this can be used to give a constraint on the mass of any stable thermal relic from the maximal cross section allowed by unitarity [15]. Using the WMAP 5-year results [32] for the most conservative upper bound for the matter density, we can update such bound. In fact imposing

$$
\Omega_{X} h^{2}=m_{X} Y_{X+\bar{X}}\left(T_{\text {now }}\right) s\left(T_{\text {now }}\right) / \rho_{c} \leq 0.13
$$

gives us for a single degree of freedom the constraint

$$
\frac{m_{X} Y_{X+\bar{X}}\left(T_{\text {now }}\right)}{\mathrm{GeV}} \leq 4.6 \times 10^{-10}
$$

resulting for a scalar particle in

$$
m_{X} \leq 280 \mathrm{TeV} .
$$

Note that for a fermionic spin $1 / 2$ relic the unitarity cross section is reduced by a factor four and therefore the bound on the mass is stronger by a factor two.

\section{Constraints on cosmological relics}

We review here the constraints on the abundance of cosmological relics that we will compare with the number density of a charged scalar relic in the next section. First we will consider the case of stable relics (i.e. with lifetimes longer than $10^{27} \mathrm{~s}$ ) and next relics 
with lifetimes in the window $0.1-10^{12} \mathrm{~s}$. Note that for shorter lifetimes the constraints are non-existent, as long as the particle did not dominate the universe dynamics before decaying or produce a large amount of entropy, while for lifetimes between $10^{10}-10^{27} \mathrm{~s}$ bounds from CMB distortion [33] and from the measured photon diffuse flux [34] apply, but will not be discussed here.

\subsection{Stable relics}

The possibility of existence of some more exotic cosmological relics than the known light elements stimulated many years ago the search for exotic nuclei in water and other materials on the earth. Those searches were unsuccessful and provide a very strong limit on the number density of any relic that would bind electromagnetically with an electron or in nuclei, under the assumption that such particles are equally distributed in the Universe compared to baryons. If such relics were present long before structure formation, it is highly probable that they were trapped together with baryons when the universe's density was still nearly homogeneous, so that we can expect their number density not to be too strongly dependent on the local environment. Note that in any case these bounds are so strong that the possibility of such a relic to be Dark Matter is completely excluded.

The most recent constraints are those obtained by [35] looking for anomalously heavy hydrogen in deep sea water, which apply to an electrically positively charged relic, and give for masses $5 \mathrm{GeV} \leq m_{X} \leq 1.6 \mathrm{TeV}$ :

$$
Y_{X^{+}} \leq 4 \times 10^{-17} Y_{B}=3.5 \times 10^{-27}\left(\frac{\Omega_{B} h^{2}}{0.0223}\right)
$$

Taking into account the gravitational effect in deep sea, this corresponds to a concentration of the order of $10^{-28}$ at sea level or equivalently

$$
Y_{X^{+}} \leq 0.9 \times 10^{-38}\left(\frac{\Omega_{B} h^{2}}{0.0223}\right),
$$

which is comparable to other limits in the same mass range, [36]. For larger masses up to a TeV, a slightly looser bound $Y_{X^{+}} / Y_{B}<3 \times 10^{-20}$ was found by [37], while for even larger masses $10 \mathrm{TeV} \leq m_{X} \leq 6 \times 10^{4} \mathrm{TeV}$ it weakens even further to $Y_{X^{+}} / Y_{B}<7 \times 10^{-15}$, as given by [38], i.e.

$$
Y_{X^{+}} \leq 6 \times 10^{-25}\left(\frac{\Omega_{B} h^{2}}{0.0223}\right)
$$

For electromagnetically neutral, but coloured relics, the bounds are obtained from considering heavier elements and are considerably weaker; using the results of [37] for Carbon, the limits are of the order $Y_{X^{+}} / Y_{B} \leq 4-8 \times 10^{-20}$ for $m_{X}=0.1-1 \mathrm{TeV}$, reaching $2 \times 10^{-16}$ at the largest mass considered $10 \mathrm{TeV}$. For larger masses $m_{X} \leq 100 \mathrm{TeV}$ only the constraint by [39] for lead is present, giving

$$
Y_{X} \leq 1.5 \times 10^{-13} Y_{B}=1.3 \times 10^{-25}\left(\frac{\Omega_{B} h^{2}}{0.0223}\right)
$$

We see that these constraints are very strong. In order to reach even the weakest bound of $Y_{X} \leq 10^{-25}$, the unitarity cross section is way too weak and needs to be increased at 
least by nine orders of magnitude, i.e.

$$
\sum_{J}(2 J+1)>10^{9}
$$

Therefore stable relics are allowed only if their interaction does not belong to the Standard Model and they cannot form exotic atoms/nuclei or if their annihilation rate becomes much larger than the unitarity one as it can happen if they interact strongly and can form intermediate bound states. But in any case, note that cross sections of the order $\pi / \Lambda_{Q C D}^{2}$ that can arise after the QCD phase transition are not sufficient to evade these constraints [31], so their interaction would have to be stronger than QCD.

\subsection{Unstable relics}

Different cosmological constraints exist on the density of an unstable relic, depending on its lifetime. For lifetimes between $0.1 \mathrm{~s}$ and $10^{10} \mathrm{~s}$, the strongest constraints come from Big Bang Nucleosynthesis. In fact, if the relic decay injects very energetic particles into the thermal bath during BBN, it can change the abundances of the light elements. Since standard BBN agrees quite well with the primordial abundances of Helium-4, Deuterium and (within a factor of two) Lithium-7 inferred from present astronomical observations [9], the relic density has to be low enough not to change those predictions too strongly. These effects are present for any decaying particle and have been studied in various papers (see 10, 40, 41, 42 and references therein). For lifetimes above $3000 \mathrm{~s}$, corresponding to the time of production of Lithium, additional constraints are present if the relic is electromagnetically charged and can form a bound state with positively charged nuclei increasing the rates for Lithium-6 production [11]. The Standard BBN prediction for the ${ }^{6} \mathrm{Li}$ abundance is actually way too small compared to the observed one, so that the presence of a charged relic with appropriate lifetime can help reconciling BBN with the measured abundances of ${ }^{6} \mathrm{Li},{ }^{7} \mathrm{Li}$ [43], but we will disregard this possibility and only concentrate on the exclusion region.

We summarise here the main results from various BBN analyses and give conservative bounds on the energy density of the decaying relic and compare them with our computation of the relic density. Since we are interested in escaping the BBN constraints, we focus mainly on the strongest bounds, but we keep conservative values for the light element abundances. Note that in many of the analysis slightly different ranges for these abundances are considered, corresponding to slightly different constraints on the decaying relic.

In general, the decay can produce very energetic SM particles that can initiate either hadronic or electromagnetic showers in the plasma. The most stringent bounds are obtained for a relic that produces mostly hadronic showers, since electromagnetic particles like photons or electrons can thermalise very quickly by interacting with the tail of the CMB distribution until times of about $10^{6} \mathrm{~s}$. So we will consider in the following the constraints for relics producing hadronic showers with a branching ratio $B_{H}=1$. We will comment later on the case where this branching ratio is smaller. There are then practically three regions of the lifetimes as discussed in [40]:

- $10^{-1} \mathrm{~s} \leq \tau \leq 10^{2} \mathrm{~s}$ : the dominant effect is the interconversion between protons and neutrons, that changes the Helium abundance, overproducing it; 
- $10^{2} \mathrm{~s} \leq \tau \leq 10^{7} \mathrm{~s}$ : hadrodissociation is the most efficient process and the bound come from the non-thermal production of $L i$ and $D$;

- $10^{7} \mathrm{~s} \leq \tau \leq 10^{12} \mathrm{~s}$ : photodissociation caused both by direct electromagnetic showers and by those generated by the daughter hadrons starts to dominate and the overproduction of ${ }^{3} \mathrm{He}$ is the main result.

It is clear that these limits depend on the decay branching ratio $B_{H}$ into hadrons for lifetimes $\tau \leq 10^{7} \mathrm{~s}$, while they are independent of $B_{H}$ for longer lifetimes. In Table 1, we give conservative bounds taken from the general analysis of [40] for the three regions, assuming $B_{H}=1$. Similar constraints were obtained independently also by [41]. Note that the bound for short lifetimes becomes approximately one order of magnitude weaker if one takes a more recent value of the ${ }^{4} \mathrm{He}$ abundance as discussed in [42. Unfortunately this new publication does not provide constraints for a general relic, but discusses only the explicit cases of a bino neutralino or a right-handed stau.

The limits we use can be parameterised as

$$
\begin{array}{cc}
Y_{X+\bar{X}} \leq 1.0 \times 10^{-13}\left(\frac{m_{X}}{1 \mathrm{TeV}}\right)^{-0.3} \quad \text { for } \tau_{X} \sim 0.1-10^{2} \mathrm{~s} \\
Y_{X+\bar{X}} \leq 1.1 \times 10^{-16}\left(\frac{m_{X}}{1 \mathrm{TeV}}\right)^{-0.57} \quad \text { for } \tau_{X} \sim 10^{2}-10^{7} \mathrm{~s} .
\end{array}
$$

The assumption $B_{H}=1$ is surely valid if the decaying relic is coloured, while $B_{H}$ can be different if it is only electromagnetically charged, as in the case of the stau. If the branching ratio into hadronic modes for the relic is less than one, the hadronic BBN bounds are relaxed accordingly by a factor $1 / B_{H}$. For intermediate lifetimes, then electromagnetic showers can become a more important effect, but only if $B_{H}<0.01$.

For electromagnetically charged relics with lifetimes longer than about $3000 \mathrm{~s}$ and low $B_{H}<0.1-0.01$, strong bounds also come from considering the catalysed overproduction of ${ }^{6} \mathrm{Li}$ [11]. In fact when bound states between nuclei and the relic can form such as ${ }^{4} \mathrm{HeX}^{-}$, many nuclear rate are modified and change the final abundance especially of ${ }^{6} \mathrm{Li}$ and ${ }^{7} \mathrm{Li}$. For particles decaying after $5 \times 10^{5} \mathrm{~s}$ it has been argued that uncertainties in the nuclear rates make such constraints weaker than the general ones discussed above [44], so we will consider here catalysed BBN constraints only for the intermediate lifetime range.

Unfortunately, different values for these bounds are given in the literature; in [45, 46] they are found to be maximally at the level of $Y_{X^{-}}<1.4-2 \times 10^{-16}$, while the latest value in [44] is maximally $Y_{X^{-}}<10^{-14}$, taking a larger window for the ratio ${ }^{6} \mathrm{Li} /{ }^{7} \mathrm{Li}$. Here we will use as a constraint the simple interpolation for the total yield 8

$$
Y_{X+\bar{X}} \leq\left\{\begin{array}{lll}
2 \times 10^{-12}\left(\frac{\tau_{X}}{3 \times 10^{3} \mathrm{~S}}\right)^{-2} & \text { for } & \tau_{X} \lesssim 10^{5} \mathrm{~s} \\
2 \times 10^{-15} & \text { for } & \tau_{X} \geq 10^{5} \mathrm{~s}
\end{array}\right.
$$

that lies somewhat in between. The bounds from catalysed BBN do not apply for coloured scalar relics because these should have a large branching ratio into hadrons, such that the 'conventional' BBN bounds from hadronic showers are much stronger. In passing note also that up-type squarks would mostly hadronise into neutral fermionic mesons which are lighter than the charged ones [30].

\footnotetext{
${ }^{8}$ The catalysed BBN constraints restrict only the abundance of the negatively charged particles, but we give here the constraint for the total yield assuming $2 Y_{X^{-}}=Y_{X+\bar{X}}$.
} 
Maximal values of $m_{X} Y_{X+\bar{X}}(\mathrm{GeV})$ allowed by BBN

\begin{tabular}{|c|c|c|c|}
\hline$m_{X}(\mathrm{TeV})$ & $10^{-1}-10^{2} \mathrm{~s}$ & $10^{2}-10^{7} \mathrm{~s}$ & $10^{7}-10^{12} \mathrm{~s}$ \\
\hline \hline 0.1 & $2 \times 10^{-11}$ & $5 \times 10^{-14}$ & $10^{-14}$ \\
\hline 1 & $1 \times 10^{-10}$ & $10^{-13}$ & $10^{-14}$ \\
\hline 10 & $5 \times 10^{-10}$ & $3 \times 10^{-13}$ & $10^{-14}$ \\
\hline \hline
\end{tabular}

Table 1: Maximal allowed values of $m_{X} Y_{X+\bar{X}}$ in the different region of lifetimes taken from Figures 38-40 of [40]. We are assuming here that the energy released in Standard Model particles is one half of $m_{X}$ as happens in a two body decay of the NLSP into LSP and the NLSP non-supersymmetric partner and that all the energy is released in hadrons. In general the strongest bound is for longer lifetimes and it is independent of $m_{X}$ and the hadronic branching ratio. The bounds in the second column come from $D$, but the ${ }^{6} \mathrm{Li}$ ones, that are sometimes considered too strong [43, are not very far away.

We summarise the constraints in Fig. 7, which shows our conservative bounds in the plane of total number density vs lifetime. Note that the constraint from catalysed BBN are for the stau stronger than the hadronic ones for lifetimes longer than $\sim 10^{4} \mathrm{~s}$ and exclude a light stau NLSP with a $100 \mathrm{GeV}$ gravitino LSP in the CMSSM [12].

Comparing with Fig. 6, we see that even for a charged relic that can annihilate efficiently, the BBN bounds are very strong; in particular the case of a simple abelian interaction seems to be excluded for any charged relic whose lifetime is longer than $0.1 \mathrm{~s}$ and produces hadronic showers with $B_{H}=1$. For the coloured case the situation is less severe, but even with the Sommerfeld enhancement, which reduces the yield substantially, it is not possible to evade the bounds completely. Still all masses above approximately $50 \mathrm{GeV}$ are excluded for lifetimes longer than $100 \mathrm{~s}$, while for shorter lifetimes masses up to $700 \mathrm{GeV}$ are allowed. A much larger number of colours than three would be needed to relax all bounds. Even the unitarity case reaches the strongest BBN constraint at masses around $700 \mathrm{GeV}$ for 3 degrees of freedom or $1 \mathrm{TeV}$ for a single one.

\section{Application to the MSSM}

Until now we have considered the ideal case that the relic particle has only one single interaction. In realistic models, however, more than one interaction - and hence more than one annihilation channel - is present, making the BBN bounds less stringent.

In this section, we discuss the concrete examples of a relic stau or stop in the MSSM. We use the MICROMEGAS package [47] to take into account all relevant annihilation and co-annihilation channels, but compare also with the results for $Y_{a b}$ or $Y_{n a b}$ for the 


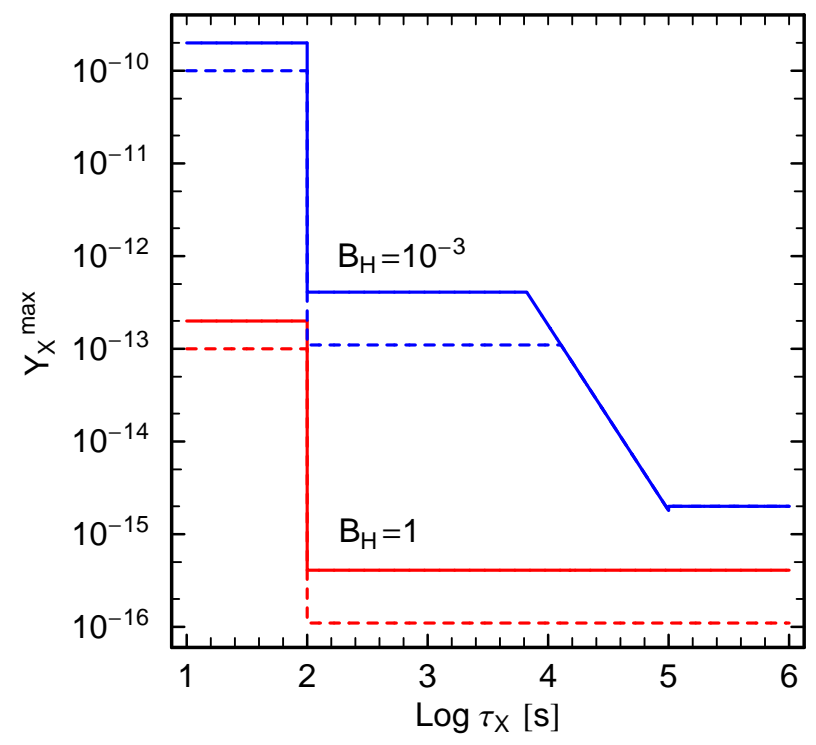

Figure 7: Maximal total yield $Y_{X+\bar{X}}^{\max }$ allowed by BBN as a function of the relic's lifetime $\tau_{X}$ for the two cases $B_{H}=1$ (red) and $B_{H}=10^{-3}$ (blue). The full lines are for a mass of $m_{X}=100 \mathrm{GeV}$, while the dashed lines are for $m_{X}=1 \mathrm{TeV}$. Note that for $B_{H}=10^{-3}$, the limit for $\tau_{X} \gtrsim 10^{4} \mathrm{~s}$ comes from $\mathrm{CBBN}$.

case of one single gauge interaction.

\subsection{Relic stau}

Our results for an electrically charged relic can be applied, for instance, to the case of the supersymmetric partner of the $\tau$. We assume here that the relic stau is a right-chiral state, $\tilde{\tau}_{R}$, and that all other SUSY particles as well as the heavy Higgs bosons decouple.

The dependence of the yield on the stau mass is shown in Fig. 8. For a $100 \mathrm{GeV}$ $\tilde{\tau}_{R}$, we get $Y_{\tilde{\tau}}=4.8 \times 10^{-13}$ at tree level from annihilation into photons (c.f. the dashed line). This is reduced by about $12 \%$ by the Sommerfeld enhancement (dashdotted line). In the full EW theory, the stau also annihilates into $W^{+} W^{-}, Z Z$ and $\gamma Z$. In fact, for $m_{\tilde{\tau}}=100 \mathrm{GeV}$, the $\gamma \gamma$ channel contributes about $55 \%, \gamma Z$ about $25 \%, Z Z$ about $10 \%$ and $W W$ about $5 \%$ to the total rate; the remaining $5 \%$ go into SM fermions. At higher stau masses, we have $\sim 50 \% \gamma \gamma$ and $\sim 30 \% \gamma Z$. Overall this gives a reduction of $Y$ by a factor of about 2 (solid line), leading to $Y_{\tilde{\tau}}=2.4 \times 10^{-13}$ at $m_{\tilde{\tau}}=100 \mathrm{GeV}$.

Staus can also annihilate into $\tau \tau$ through $t$-channel neutralino exchange. We here consider only the bino contribution. Lowering the bino mass $m_{\tilde{B}}$ decreases the yield until bino-stau coannihilation takes over, increasing it again. We find a minimum yield at about $m_{\tilde{B}} \simeq(1.1-1.2) m_{\tilde{\tau}_{1}}$, shown as dotted line in Fig. 8, It is roughly a factor 2 lower than the solid line, in agreement with [25]. Note also that the neutralino exchange leads to annihilation of same-sign stau pairs, $\tilde{\tau}_{1}^{ \pm} \tilde{\tau}_{1}^{ \pm} \rightarrow \tau^{ \pm} \tau^{ \pm}$, so this process gets Sommerfeldsuppressed, and the total Sommerfeld effect almost cancels.

The annihilation into $W^{+} W^{-}$and $Z Z$ is considerably enhanced if the relic stau also has some $\tilde{\tau}_{L}$ component, $\tilde{\tau}_{1}=\tilde{\tau}_{R} \sin \theta+\tilde{\tau}_{L} \cos \theta$ with $\cos \theta \neq 0$. In this case also t-channel exchange of $\tilde{\nu}_{\tau}$ (for $W^{+} W^{-}$) and $\tilde{\tau}_{2}$ (for $Z Z$ ) has to be taken into account in addition 


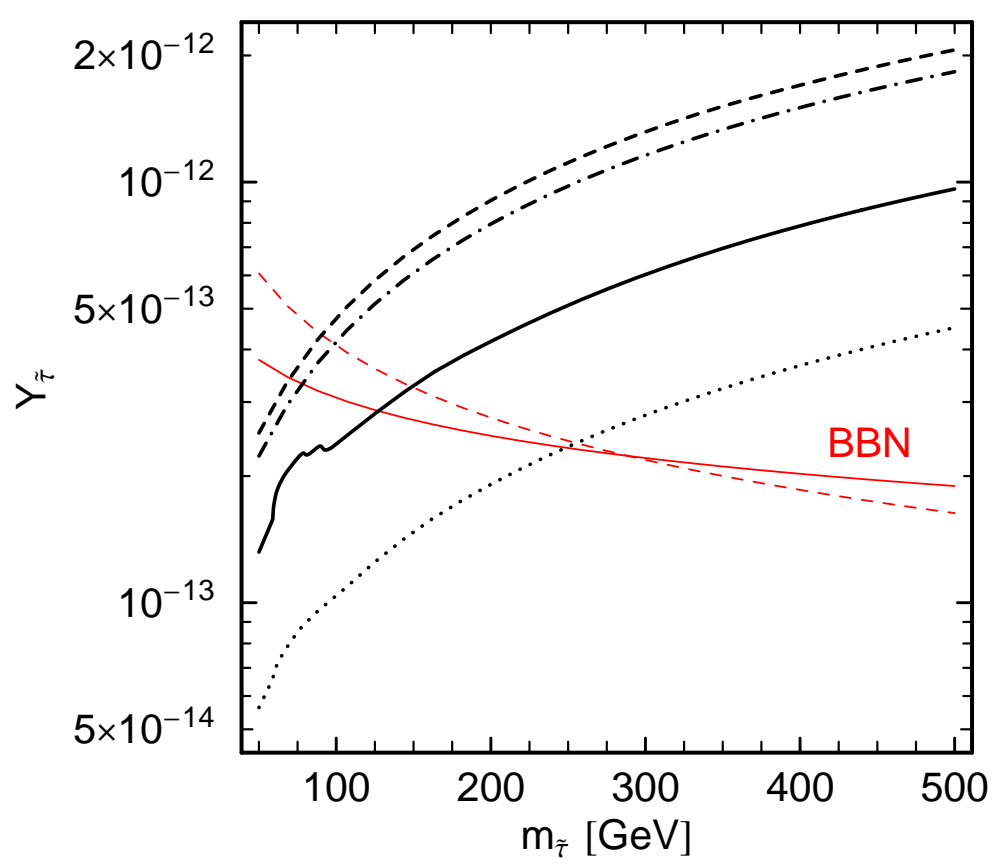

Figure 8: Total yield $Y$ (black lines) for a relic $\tilde{\tau}_{R}$ as function of the stau mass. The dashed and dashdotted curves are tree-level and Sommerfeld- corrected results, respectively, from annihilation into photons (i.e. $Y_{a b}$ ). The full line includes also annihilation into $W$ and $Z$ bosons, assuming all other sparticles decouple. Finally, the dotted line shows the case $m_{\tilde{B}}=1.1 m_{\tilde{\tau}_{1}}$. BBN bounds are shown in red: as full line for $0.1-100 \mathrm{~s}$ lifetime and $B_{H}=0.65$, and as dashed line for $>100$ s lifetime and $B_{H}=10^{-3}$. Note that if the lifetime exceeds about $10^{4} \mathrm{~s}$, the CBBN constraints become more important and quickly exclude number densities at the level of $10^{-13}-10^{-15}$, see Fig. 7 .

to the 4 -vertex and $s$-channel $\gamma / Z$ exchange, c.f. Appendix B. It turns out that these $t$-channel diagrams lead to a destructive interference: for given $\cos \theta$, smaller $\tilde{\nu}_{\tau}$ and $\tilde{\tau}_{2}$ masses lead to smaller cross sections. Since the stau and sneutrino masses and stau mixing angle are related to each other, one cannot simply maximise the cross section by choosing maximal mixing $(\cos \theta=0.7)$ and very heavy $\tilde{\nu}_{\tau}$ and $\widetilde{\tau}_{2}$. However, for reasonable parameter choices, it is still possible to reduce the yield shown in Fig. 8 by up to about an order of magnitude.Alternatively, one could rely on resonant annihilation through s-channel Higgs exchange or on coannihilation with sparticles that are close in mass to bring $Y_{\tilde{\tau}}$ below the BBN bounds.

Barring these possibilities of largely enhanced cross sections, the stau lifetime and branching ratio into hadronic modes become key parameters to decide whether the scenario is allowed. First of all, let us discuss briefly the branching ratio into hadrons. We are considering here the decay $\tilde{\tau}_{R} \rightarrow \tau+\mathrm{LSP}$. The $\tau$ decays into charged mesons $65 \%$ of the time, while the remaining times into leptons only. Charged mesons have a similar effect as nucleons during BBN only at short times $<100$ seconds, because later they decay before interacting with nucleons and give rise only to electromagnetic showers [40]. Therefore we will take $B_{H}(\tilde{\tau}) \sim 0.65$ for lifetimes up to $100 \mathrm{~s}$, while it becomes much smaller for longer lifetimes, we will use $B_{H}(\tilde{\tau}) \sim 10^{-3}$ as reference value. This is in the 

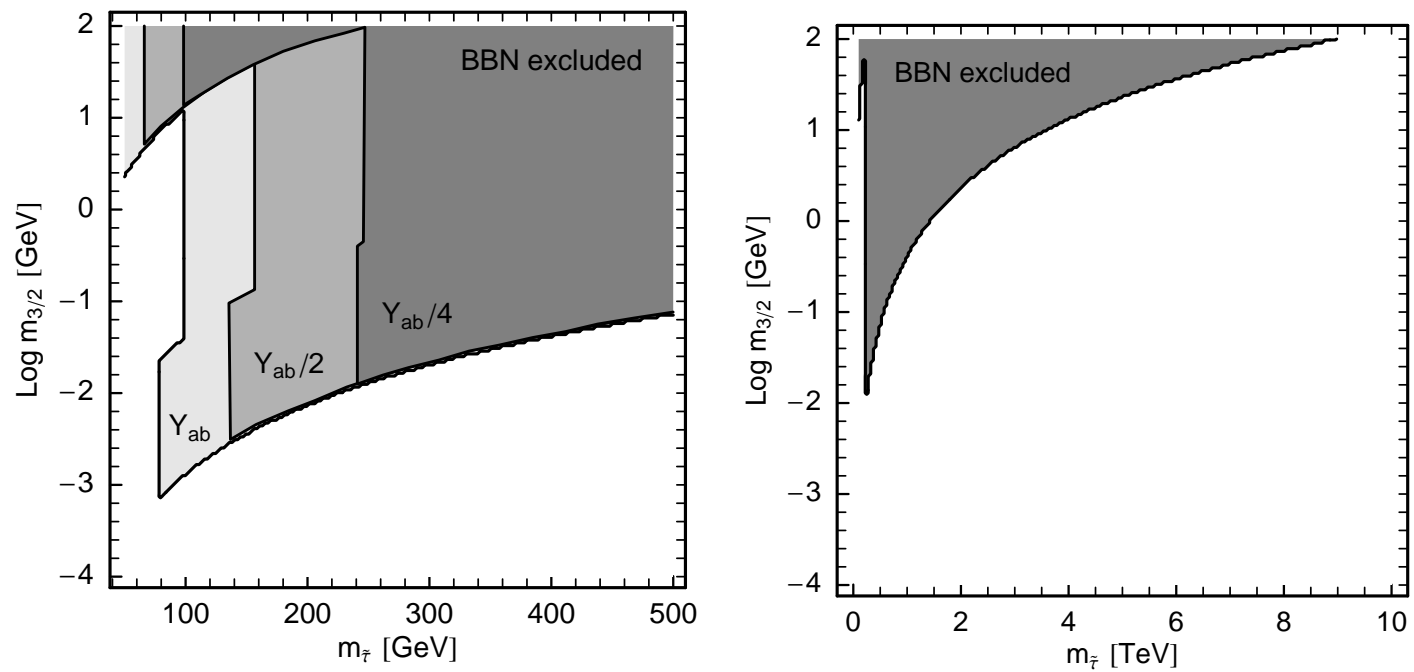

Figure 9: BBN-excluded regions for a gravitino LSP in the plane $m_{3 / 2}$ vs. $m_{\tilde{\tau}}$. On the left a zoom on $m_{\tilde{\tau}}=50-500 \mathrm{GeV}$ for $Y_{\tilde{\tau}}=Y_{a b}$ (light grey ), $Y_{\tilde{\tau}}=Y_{a b} / 2$ (medium grey) and $Y_{\tilde{\tau}}=Y_{a b} / 4$ (dark grey). On the right for $m_{\tilde{\tau}}=0.1-10 \mathrm{TeV}$. Note that LEP excluded $m_{\tilde{\tau}} \leq 99.4 \mathrm{GeV}$ for a charged particle stable within the detector [50].

central range computed recently for the stau decay into tau, gravitino and a $q \bar{q}$ pair, and we refer to that result for a more detailed analysis [48]. (A full computation including a more complete treatment of the hadronic decays of the tau for the case of a right-handed stau has been given in [42].) We have then to apply the BBN bounds discussed in the previous section corrected by these branching ratio factors, according to the time of decay.

Regarding the stau lifetime, this depends strongly on the nature of the LSP. For the case of the axino LSP, the decay rate is given by

$$
\Gamma\left(\tilde{\tau}_{R} \rightarrow \tau \tilde{a}\right)=(25 \mathrm{~s})^{-1} \xi^{2}\left(\frac{m_{\tilde{\tau}}}{10^{2} \mathrm{GeV}}\right)\left(\frac{m_{\tilde{B}}}{10^{2} \mathrm{GeV}}\right)^{2}\left(\frac{10^{11} \mathrm{GeV}}{f_{a}}\right)^{2}\left(1-\frac{m_{\tilde{a}}^{2}}{m_{\tilde{\tau}}^{2}}\right)
$$

where $m_{\tilde{a}}$ is the axino mass, $m_{\tilde{B}}$ is the Bino mass, $f_{a}$ is the Peccei-Quinn scale, and $\xi$ is a factor of order 1 taking into account some uncertainties in the loop computation [49]. Therefore only the weakest BBN bound applies and actually disappears completely for large stau mass: in fact even for the conservative case $m_{\tilde{B}}=1.1 m_{\tilde{\tau}}$ and $f_{a}=10^{11} \mathrm{GeV}$, the lifetime becomes shorter than $0.1 \mathrm{~s}$ for $m_{\tilde{\tau}} \leq 590 \mathrm{GeV}$. We are here neglecting the case of a strong degeneracy between the stau and axino masses. We see therefore that for axino LSP a very light stau is a viable possibility and, depending on the supersymmetric spectrum, only the mass window between $125 / 250-590 \mathrm{GeV}$ is possibly excluded by the BBN constraints, as can be seen from Fig. 8. In that region however probably a more proper computation of the stau hadronic branching ratio and its effect in the early stages of $\mathrm{BBN}$ is needed, as discussed in [42]. In fact comparing our exclusion region with theirs, we find that their constraints are much weaker for short lifetimes, due to an up-dated value of the Helium abundance and a larger systematic error, allowing all the stau region for an axino LSP. 
For a gravitino LSP, the decay rate is given by [49]

$$
\Gamma\left(\tilde{\tau}_{R} \rightarrow \tau \tilde{G}\right)=\left(5.9 \times 10^{8} \mathrm{~s}\right)^{-1}\left(\frac{m_{\tilde{\tau}}}{100 \mathrm{GeV}}\right)^{5}\left(\frac{100 \mathrm{GeV}}{m_{3 / 2}}\right)^{2}\left(1-\frac{m_{3 / 2}^{2}}{m_{\tilde{\tau}}^{2}}\right)^{4}
$$

which typically gives longer stau lifetimes than the axino case. Figure 9 shows the BBNexcluded region in the $m_{3 / 2}$ vs $m_{\tilde{\tau}}$ plane. We consider a number density $Y_{\tilde{\tau}}$ equal to $1 / 2$ and $1 / 4$ times $Y_{a b}$ to account for the possible variation depending on $m_{\tilde{B}}$. As can be seen, to avoid all bounds we need either a very light gravitino in the MeV range for $m_{\tilde{\tau}} \sim \mathcal{O}(100) \mathrm{GeV}$, or a very heavy stau, e.g. $m_{\tilde{\tau}} \gtrsim 1.4 \mathrm{TeV}(9 \mathrm{TeV})$ for $m_{3 / 2}=1 \mathrm{GeV}$ $(100 \mathrm{GeV})$, corresponding to a stau lifetime shorter than $0.1 \mathrm{~s}$. On the other hand, for $m_{\tilde{\tau}} \sim 100-250 \mathrm{GeV}$ and a lifetime longer than $100 \mathrm{~s}, B_{H} \sim 10^{-3}$ can bring the effective yield below the bound of $m Y \approx 5 \times 10^{-14}$ required by hadronic showers. Last but not least, note that the constraint from catalysed BBN becomes stronger than the hadronic ones for lifetimes longer than about $10^{4} \mathrm{~s}$ and excludes a light stau NLSP for gravitino masses above 10-100 GeV.

\subsection{Relic stop}

To discuss the case of a relic stop, we assume that only $\tilde{t}_{R}$ is light while all other SUSY particles are heavy and decouple. Moreover, we assume that the light Higgs is SM-like with a mass of $m_{h}=115 \mathrm{GeV}$, and that the other Higgs bosons are also heavy and do not contribute to the stop annihilation.

Results for the yield as a function of the stop mass are shown in Fig. 10, Let us first discuss the left plot, Fig. 10(a), which shows the yield at leading order (LO). Here the full line is the pure QCD result, $Y_{n a b}$ for $S U(3)$, without Sommerfeld correction. As can be seen, $\tilde{t} \tilde{t}^{*} \rightarrow g g$ alone is efficient enough to avoid the BBN constraints up to stop masses of about $700 \mathrm{GeV}$. In the full theory, the stop can also annihilate into other particles, in particular into EW gauge and Higgs bosons. The yield for the QCD+EW case, still assuming heavy sparticles, is shown as the dashed line in Fig. 10(a). The dip at $m_{\tilde{t}} \sim 120$ $\mathrm{GeV}$ is due to the onset of $\tilde{t}_{R} \tilde{t}_{R}^{*} \rightarrow h h$. Other important channels are annihilation into $W^{+} W^{-}$and $\gamma g$, contributing about $10 \%$ each to the total annihilation cross section for $m_{\tilde{t}} \gtrsim 200 \mathrm{GeV}$. Annihilation into $Z Z$ contributes about $5 \%$. Annihilation into top quarks is suppressed by the heavy gluino mass, and also by $m_{t}$. However, if $m_{\tilde{t}}>200 \mathrm{GeV}$ and $m_{\tilde{g}} \sim 2 m_{\tilde{t}}, \tilde{t}_{R} \tilde{t}_{R} \rightarrow t t$ further reduces the yield by 10-20\%. This is shown as the dash-dotted line in Fig. 10(a). All in all, annihilation into gluons is, however, always the dominant channel, contributing at least $50 \%$. We therefore take $Y_{n a b} / 2$ as a rough limit, which is shown as the dotted line in Fig. 10(a). Comparing with the BBN constraints we see that a relic $\tilde{t}_{R}$ with a lifetime of $0.1-100 \mathrm{~s}$ can be in agreement with BBN even for high masses of about $1 \mathrm{TeV}$.

The impact of the Sommerfeld enhancement is illustrated in Fig. 10(b) for the case

$\tilde{t} \tilde{t}^{*} \rightarrow g g$. As can be seen, taking the averaged Sommerfeld factor of $C_{S U(3)}^{a v}=11 / 42$ in eq. (29) reduces the LO yield by roughly a factor of 2 , while a summed factor according to eq. (34) reduces the LO yield by roughly a factor of 3 . These results are in qualitative agreement with those of [20], that considered the Sommerfeld correction in the neutralinostop coannihilation region. Here note that for colour-singlet channels like, for instance, $\tilde{t} \tilde{t}^{*} \rightarrow W^{+} W^{-}$a factor of $C=4 / 3$ applies, hence leading to even larger enhancement. We 

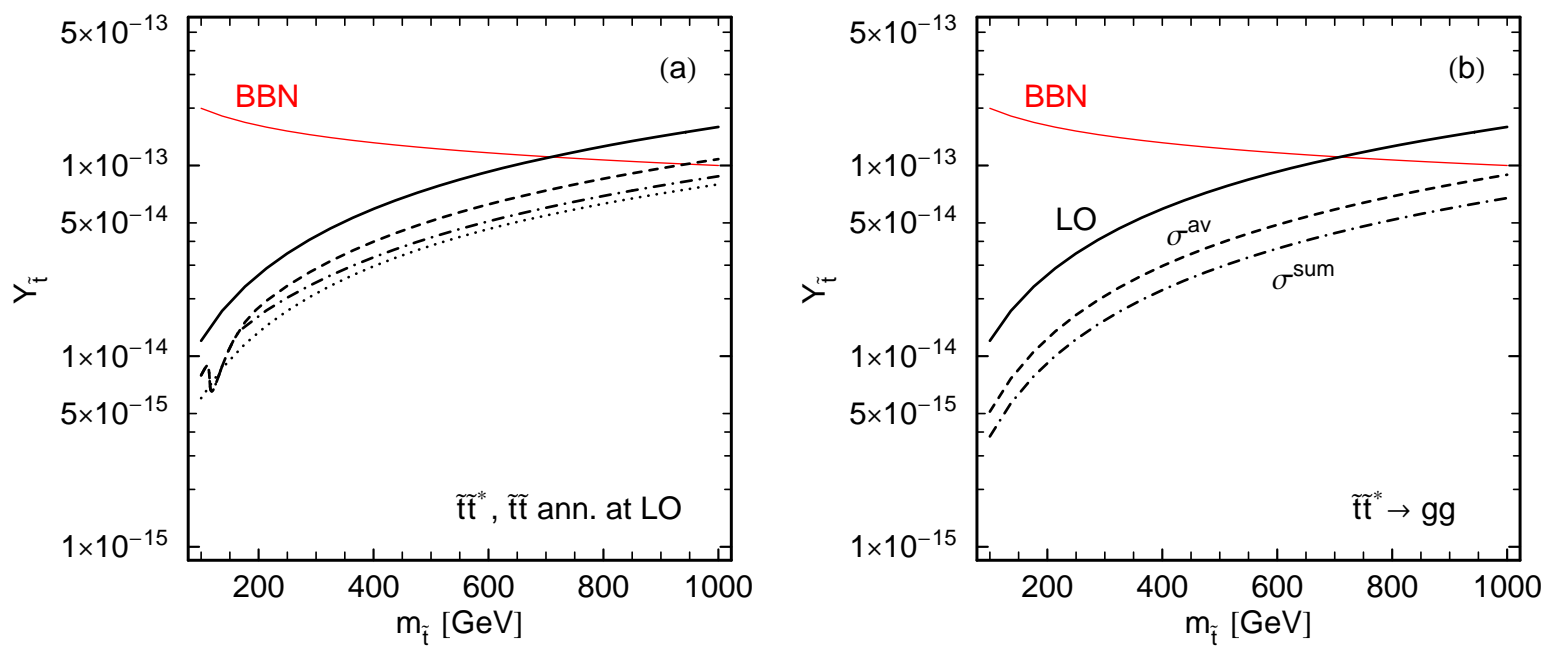

Figure 10: Results for $Y_{\tilde{t}}$ for a relic $\tilde{t}_{R}$ as a function of the stop mass. In (a), tree-level results for different channels: the solid line comes from $\tilde{t} \tilde{t}^{*} \rightarrow g g$ only, the dashed line includes all channels into QCD+EW gauge and $h$ bosons (case of decoupled sparticles and heavy Higgses), the dash-dotted line is the result for $m_{\tilde{g}}=2 m_{\tilde{t}_{R}}$, and the dotted line the limit $Y_{\tilde{t}}=Y_{n a b} / 2$. In (b), the effect of the Sommerfeld enhancement on the yield from $\tilde{t} \tilde{t}^{*} \rightarrow g g$ : the full line shows the tree level result, the dashed line the result for $\sigma^{\text {SFav }}$, i.e. applying an averaged Sommerfeld factor $C_{S U(3)}^{\text {av }}=11 / 42$, and the dash-dotted line is for $\sigma^{\text {SFsum }}$, i.e. applying a summed factor according to eq. (34). The BBN bound for $0.1-100 \mathrm{~s}$ lifetime is shown as thin red line in both plots.

leave a detailed numerical analysis of the enhancement of the various stop annihilation channels for future work. Here we just note that the overall effect can be a reduction of the yield by an order of magnitude.

Additional annihilation can take place after the QCD phase transition, when the stops are in a confined phase with the quarks. Since the lighter fermionic mesons are neutral and assuming that the annihilation process takes place without the formation of a bound state between the mesinos, the unitarity cross section is probably a good estimate of such annihilation and allows for heavier stops to be consistent with hadronic shower constraints. We see in fact from Fig. 6 that the unitary cross section with three degrees of freedom gives a yield well below all the BBN bounds (and below the range in Fig. 10) for stop masses up to $700 \mathrm{GeV}$. If also bound states between the mesinos can form efficiently, the BBN constraints disappear altogether [31, but note that we do not have to rely on the enhancement coming from such processes, which are very difficult to compute, for a wide range of parameter space.

Let us briefly discuss the lifetime also for the stop case. For the case of an axino LSP, the stop decay rate is a larger than for the stau since it depends on the gluino mass and the QCD gauge coupling [3]:

$$
\Gamma\left(\tilde{t}_{R} \rightarrow t \tilde{a}\right)=\left(1.3 \times 10^{-3} \mathrm{sec}\right)^{-1} \xi_{t}^{2}\left(\frac{m_{\tilde{t}}}{10^{2} \mathrm{GeV}}\right)\left(\frac{m_{\tilde{g}}}{10^{2} \mathrm{GeV}}\right)^{2}\left(\frac{10^{11} \mathrm{GeV}}{f_{a}}\right)^{2}\left(1-\frac{m_{\tilde{a}}^{2}}{m_{\tilde{t}}^{2}}\right)
$$

where $\xi_{t}$ is again a factor of order one taking into account the uncertainties in the loop 


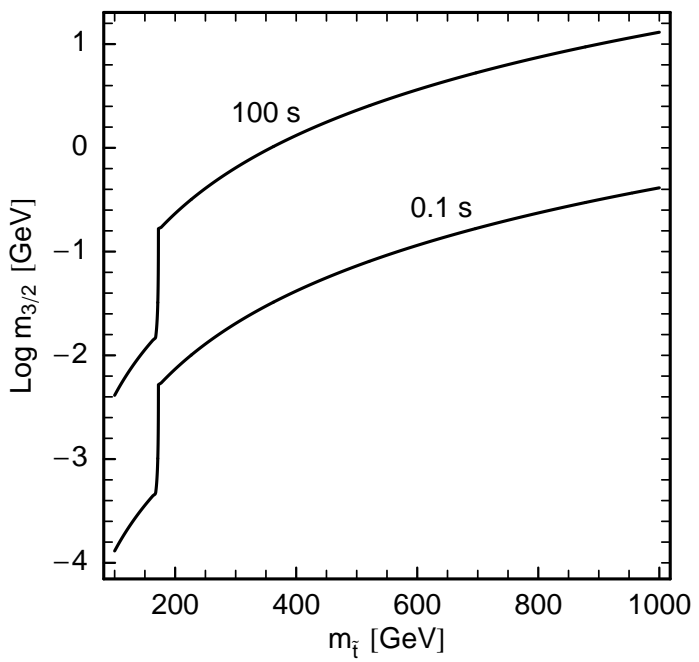

Figure 11: Lifetime of a relic $\tilde{t}_{R}$ in the plane $m_{3 / 2}$ vs $m_{\tilde{t}}$. Recent results from the Tevatron exclude a metastable stop below $250 \mathrm{GeV}[52$.

computation [49], in principle different than the one for the stau. Therefore, for the axino case, the BBN bound never applies if the decay into top is kinematically allowed, i.e. if $m_{\tilde{t}}^{2} \geq\left(m_{\tilde{a}}+m_{t}\right)^{2}$. If the stop mass is smaller, the decay can proceed through a virtual top, for which we estimate a suppression of order $\mathcal{O}(1 / 100)$ due to the 3-body phase space. This would still give a lifetime of order $0.1 \mathrm{sec}$, so the BBN constraints are completely avoided, as long as there is not a strong degeneracy in mass between LSP and NLSP or the factor $\xi_{t}$ is exceptionally large.

For a gravitino LSP, on the other hand, the same formula applies for stop as for stau, eq. (60) with $\tilde{\tau} \rightarrow \tilde{t}$, because the gravitino couples only to mass. Note, however, that also in this decay the width gets phase-space suppressed if $m_{\tilde{t}}<m_{t}+m_{\tilde{G}}$. For illustration, we show in Fig. 11 the band of 0.1-100 s lifetime in the plane $m_{3 / 2}$ vs $m_{\tilde{t}}$. For lifetimes longer than $100 \mathrm{~s}$, stops can still be in accord with BBN thanks to the additional annihilation during the QCD phase transition, if their annihilation reaches the unitarity one. We therefore conclude that cosmologically stops are an allowed NLSP in any mass range and in particular also for a heavy gravitino. Our results are in agreement with those for specific supersymmetric models with stop NLSP discussed in [51]. From the colliders side, note that the low mass region $m_{\tilde{t}}<250 \mathrm{GeV}$ has been recently excluded by the search for charged massive particles at the Tevatron [52].

\section{Conclusions}

We have studied the number density of a charged relic by computing the annihilation cross section into gauge bosons, including the Sommerfeld enhancement. We have found that the Sommerfeld factor increases the thermally averaged annihilation cross section by $20-50 \%$ and reduces the final yield even by a factor 2 or 3 for the $S U(3)$ case. Moreover the result is very sensitive on how the higher orders are resummed. Nevertheless the number density surviving the annihilation is still large and BBN constraints are relevant for most relics. They can be avoided completely only for very large $N$ for particles in the 
fundamental representation of $S U(N)\left(N>100\right.$ for $\left.m_{X} \leq 10 \mathrm{TeV}\right)$ or for cross sections nearly fulfilling the unitarity bound. For the cases of SM gauge groups, the allowed regions only correspond to very light relic masses, where the number density is low enough, or to sufficiently heavy relic masses so that the decay takes place in the first stages of BBN. The latter allowed region depends strongly on the relic decay channel, and, in case of a gravitino LSP with conserved R-parity, also on the gravitino mass. Let us mention here that if R-parity is just marginally broken, the NLSP can decay with shorter lifetime through R-parity violating channels and the BBN constraints can be easily evaded for any NLSP while keeping the gravitino LSP as Dark Matter [53].

More specifically, for the stau NLSP the light mass window has nearly completely been excluded by direct searches at LEP, even if the annihilation cross-section is maximal $4 \sigma\left(\tilde{\tau} \tilde{\tau}^{*} \rightarrow \gamma \gamma\right)$, unless the gravitino is lighter than a few tens of $\mathrm{GeV}$, while the large mass region is unfortunately out of reach at the LHC for gravitino masses $m_{3 / 2}>100 \mathrm{GeV}$. The detection of a quasi-stable stau at the LHC would then point to a scenario with relatively light gravitino mass, R-parity breaking or an axino LSP and could probably exclude the gravity mediated supersymmetry breaking scenario. In that case the determination of the stau lifetime and its decays will become crucial in distinguishing the different LSPs [7, 49].

The stop case is much less constrained thanks to the stronger annihilation crosssection, even if in this case the decay always produces mainly hadrons. We have practically no constraints if the LSP is an axino and even for a gravitino LSP, we can allow for relatively light stops up to approximately $700 \mathrm{GeV}\left(1 \mathrm{TeV}\right.$ for lifetimes below $10^{7} \mathrm{~s}$ ), if the annihilation cross section reaches the unitarity one after the QCD phase transition. The window between the present Tevatron bound around $250 \mathrm{GeV}$ and $1 \mathrm{TeV}$ should be surely completely covered by the LHC, the signature being a quasi-stable heavy fermionic meson. The detection of such a state would call for a non-minimal SUSY breaking sector with a coloured NLSP and a very weakly interacting LSP. In this case again only the analysis of the stop decays would allow to distinguish between the lightest states.

\section{Acknowledgements}

We thank Nans Baro, Fawzi Boudjema, Lance Dixon, Koichi Hamaguchi, Wolfgang Kilian, Tom Rizzo, Masato Senami and Iain Stewart for useful discussions and communications. We thank specially Alexander Pukhov for valuable help with implementing the Sommerfeld enhancement in Micromegas.

CFB's work is supported in part by funds provided by the U.S. Department of Energy (D.O.E.) under cooperative research agreement DE-FC02-94ER40818 and by the National Science Foundation under Grant No. PHY05-51164.

LC would like to thank the Institute of Theoretical Physics of the Warsaw University for their hospitality during part of this work; the visit was supported by a Maria Curie Transfer of Knowledge Fellowship of the European Community's Sixth Framework Programme under contract number MTKD-CT-2005-029466 (2006-2010). LC also acknowledges the support of the "Impuls- und Vernetzungsfond" of the Helmholtz Association under the contract number VH-NG-006 and of the European Network of Theoretical Astroparticle Physics ILIAS/N6 under contract number RII3-CT-2004-506222.

This work is also part of the French ANR project ToolsDMColl, BLAN07-2-194882. 


\section{A Annihilation into massless $S U(N)$ gauge bosons}

\section{A.1 Amplitudes for the annihilation}

We consider the case of one particle and antiparticle in the representation $T_{i}^{a}$ and its conjugate, with momenta $p_{1}, p_{2}$ and mass $m$ annihilating into two massless gauge bosons with group indices $a, b$, momenta $p_{3}, p_{4}$ and Lorentz indices $\mu, \nu$ respectively.

The process has four different contributions, corresponding to the following four Feynman diagrams:

t particle exchange in the t-channel described by the amplitude

$$
\mathcal{A}_{t}^{\mu \nu}=i g_{N}^{2}\left(T^{b} T^{a}\right)_{j i} \frac{\left(2 p_{1}-p_{3}\right)^{\mu}\left(2 p_{2}-p_{4}\right)^{\nu}}{t-m^{2}} ;
$$

$\mathbf{u}$ particle exchange in the $\mathrm{u}$-channel described by the amplitude

$$
\mathcal{A}_{u}^{\mu \nu}=i g_{N}^{2}\left(T^{a} T^{b}\right)_{j i} \frac{\left(2 p_{1}-p_{4}\right)^{\nu}\left(2 p_{2}-p_{3}\right)^{\mu}}{u-m^{2}}
$$

note that this contribution is identical to the t-channel under interchange of $a \leftrightarrow b$, $\mu \leftrightarrow \nu,\left(p_{3}, t\right) \leftrightarrow\left(p_{4}, u\right)$

4 supersymmetric four-scalar coupling giving the amplitude:

$$
\mathcal{A}_{4}^{\mu \nu}=i g_{N}^{2}\left\{T^{a}, T^{b}\right\}_{j i} g^{\mu \nu}
$$

this contribution is symmetric in the exchange of $a, b$ and therefore also $\mu, \nu$;

s off-shell gauge boson in the s-channel decaying into two bosons via the non-abelian interaction:

$$
\begin{aligned}
\mathcal{A}_{s}^{\mu \nu}= & -i g_{N}^{2}\left[T^{a}, T^{b}\right]_{j i} \frac{1}{s}\left[g^{\mu \nu}(t-u)-\left(2 p_{4}+p_{3}\right)^{\mu}\left(p_{1}-p_{2}\right)^{\nu}\right. \\
& \left.+\left(p_{1}-p_{2}\right)^{\mu}\left(2 p_{3}+p_{4}\right)^{\nu}\right]
\end{aligned}
$$

this contribution is completely antisymmetric under the exchange of the gauge bosons group indices and therefore also under the exchange of their momenta and Lorentz indices.

For convenience, we can then separate the amplitude into symmetric and antisymmetric part in colours $a, b$; then the interference between the two parts vanishes. Using

$$
T^{a} T^{b}=\frac{1}{2}\left\{T^{a}, T^{b}\right\}+\frac{1}{2}\left[T^{a}, T^{b}\right]
$$

we have then

$$
\mathcal{A}_{\text {sym }}^{\mu \nu}=\frac{i g_{N}^{2}}{2}\left\{T^{a}, T^{b}\right\}_{j i}\left[\frac{\left(2 p_{1}-p_{3}\right)^{\mu}\left(2 p_{2}-p_{4}\right)^{\nu}}{t-m^{2}}+\frac{\left(2 p_{1}-p_{4}\right)^{\nu}\left(2 p_{2}-p_{3}\right)^{\mu}}{u-m^{2}}+2 g^{\mu \nu}\right]
$$


and

$$
\begin{aligned}
\mathcal{A}_{\text {asym }}^{\mu \nu}= & \frac{i g_{N}^{2}}{2}\left[T^{a}, T^{b}\right]_{j i}\left[-\frac{\left(2 p_{1}-p_{3}\right)^{\mu}\left(2 p_{2}-p_{4}\right)^{\nu}}{t-m^{2}}+\frac{\left(2 p_{1}-p_{4}\right)^{\nu}\left(2 p_{2}-p_{3}\right)^{\mu}}{u-m^{2}}\right. \\
& \left.-2 \frac{g^{\mu \nu}(t-u)-\left(2 p_{4}+p_{3}\right)^{\mu}\left(p_{1}-p_{2}\right)^{\nu}+\left(p_{1}-p_{2}\right)^{\mu}\left(2 p_{3}+p_{4}\right)^{\nu}}{s}\right] .
\end{aligned}
$$

In the Boltzmann equation, we have to insert the averaged cross-section, so first we have to sum over all the final and initial states, i.e. sum over the gauge bosons polarisations and over all the group indices.

\section{A.2 The matrix element}

The computation for the symmetric piece is straightforward:

$$
\begin{aligned}
\left|\mathcal{A}_{\text {sym }}\right|^{2}= & g_{N}^{4}\left|\left\{T^{a}, T^{b}\right\}_{j i}\right|^{2}\left[\frac{\left(t+m^{2}\right)^{2}}{\left(t-m^{2}\right)^{2}}+\frac{\left(u+m^{2}\right)^{2}}{\left(u-m^{2}\right)^{2}}+\frac{1}{2} \frac{\left(s-4 m^{2}\right)^{2}}{\left(t-m^{2}\right)\left(u-m^{2}\right)}\right. \\
& \left.+4+\frac{s / 2-4 m^{2}-2\left(t-m^{2}\right)}{t-m^{2}}+\frac{s / 2-4 m^{2}-2\left(u-m^{2}\right)}{u-m^{2}}\right] \\
= & 4 g_{N}^{4}\left|\left\{T^{a}, T^{b}\right\}_{j i}\right|^{2}\left[\frac{1}{2}+\frac{2 m^{4}}{\left(t-m^{2}\right)^{2}}+\frac{2 m^{2}}{t-m^{2}}\left(1-\frac{2 m^{2}}{s}\right)\right] .
\end{aligned}
$$

In the antisymmetric part instead we have to take into account ghost subtraction and the total result is

$$
\begin{aligned}
\left|\mathcal{A}_{\text {asym }}\right|^{2}= & g_{N}^{4}\left|\left[T^{a}, T^{b}\right]_{j i}\right|^{2}\left[\frac{\left(t+m^{2}\right)^{2}}{\left(t-m^{2}\right)^{2}}+\frac{\left(u+m^{2}\right)^{2}}{\left(u-m^{2}\right)^{2}}-4\right. \\
& -\frac{1}{2} \frac{\left(s-4 m^{2}\right)^{2}}{\left(t-m^{2}\right)\left(u-m^{2}\right)}+2 \frac{(t-u)^{2}}{s^{2}}+\frac{16 m^{2}}{s} \\
& +\frac{(t-u)\left(3 / 2 s-t-3 m^{2}\right)+2\left(s-4 m^{2}\right)\left(u-m^{2}\right)}{s\left(t-m^{2}\right)} \\
& \left.+\frac{(u-t)\left(3 / 2 s-u-3 m^{2}\right)+2\left(s-4 m^{2}\right)\left(t-m^{2}\right)}{s\left(u-m^{2}\right)}\right] \\
= & 4 g_{N}^{4}\left|\left[T^{a}, T^{b}\right]_{j i}\right|^{2}\left[\frac{(t-u)^{2}}{2 s^{2}}+\frac{4 m^{2}}{s}+\frac{2 m^{4}}{\left(t-m^{2}\right)^{2}}\right. \\
& \left.+\frac{2 m^{2}}{t-m^{2}}\left(1+\frac{2 m^{2}}{s}\right)\right] .
\end{aligned}
$$

So for the total matrix element we have

$$
\begin{aligned}
|\mathcal{M}|^{2}= & 4 g_{N}^{4}\left\{\left|\left\{T^{a}, T^{b}\right\}_{j i}\right|^{2}\left[\frac{1}{2}+\frac{2 m_{X}^{4}}{\left(t-m_{X}^{2}\right)^{2}}+\frac{2 m_{X}^{2}}{t-m_{X}^{2}}\left(1-\frac{2 m_{X}^{2}}{s}\right)\right]\right. \\
& +\left|\left[T^{a}, T^{b}\right]_{j i}\right|^{2}\left[\frac{1}{2} \frac{\left(s+2\left(t-m_{X}^{2}\right)\right)^{2}}{s^{2}}+\frac{4 m_{X}^{2}}{s}+\frac{2 m_{X}^{4}}{\left(t-m_{X}^{2}\right)^{2}}\right. \\
& \left.\left.+\frac{2 m_{X}^{2}}{t-m_{X}^{2}}\left(1+\frac{2 m_{X}^{2}}{s}\right)\right]\right\} .
\end{aligned}
$$

and the cross section is given in eq. (16). 


\section{A.3 Comparison with QCD result}

For the case of $S U(3)$ we have

$$
\sum_{a, b, i, j}\left|\left\{T^{a}, T^{b}\right\}_{j i}\right|^{2}=\frac{28}{3}
$$

and

$$
\sum_{a, b, i, j}\left|\left[T^{a}, T^{b}\right]_{j i}\right|^{2}=\frac{1}{2} \sum_{a, b, c} f_{a b c}^{2}=12 .
$$

So after the sum over colours, we get

$$
\begin{aligned}
|\mathcal{M}|^{2}= & 4 g_{3}^{4}\left[\frac{14}{3}+6 \frac{(t-u)^{2}}{s^{2}}+\frac{48 m^{2}}{s}+\frac{2 m^{4}}{\left(t-m^{2}\right)^{2}}\left(\frac{28}{3}+12\right)\right. \\
& \left.+\frac{2 m^{2}}{t-m^{2}}\left(\frac{28}{3}+12+\frac{2 m^{2}}{s}\left(-\frac{28}{3}+12\right)\right)\right] \\
= & 4 g_{3}^{4}\left[\frac{32}{3}+24 \frac{t-m^{2}}{s}+24 \frac{\left(t-m^{2}\right)^{2}}{s^{2}}+\frac{48 m^{2}}{s}\right. \\
& \left.+\frac{128}{3} \frac{m^{4}}{\left(t-m^{2}\right)^{2}}+\frac{128}{3} \frac{m^{2}}{t-m^{2}}\left(1+\frac{1}{4} \frac{m^{2}}{s}\right)\right] .
\end{aligned}
$$

This result coincides with the one given in the literature for the QCD case [26]. Compare in general with [26]:

$$
\begin{aligned}
|\mathcal{M}(g g \rightarrow \tilde{q} \overline{\tilde{q}})|^{2}= & 4 n_{f} g_{3}^{4}\left[C_{0}\left(1-2 \frac{\left(t-m^{2}\right)\left(u-m^{2}\right)}{s^{2}}\right)-C_{K}\right] \times \\
& \times\left[1-2 \frac{s m^{2}}{\left(t-m^{2}\right)\left(u-m^{2}\right)}\left(1-\frac{s m^{2}}{\left(t-m^{2}\right)\left(u-m^{2}\right)}\right)\right] \\
= & 4 n_{f} g_{3}^{4}\left[C_{0}-C_{K}+2 C_{0} \frac{t-m^{2}}{s}+2 C_{0} \frac{\left(t-m^{2}\right)^{2}}{s^{2}}\right. \\
& +4 C_{0} \frac{m^{2}}{s}+4\left(C_{0}-C_{K}\right) \frac{m^{4}}{\left(t-m^{2}\right)^{2}} \\
& \left.+4 \frac{m^{2}}{t-m^{2}}\left(C_{0}-C_{K}+2 C_{K} \frac{m^{2}}{s}\right)\right]
\end{aligned}
$$

using again the symmetry in $u \leftrightarrow t$ and eliminating $u$.

We have also that

$$
C_{0}=\sum_{a, b, c} f_{a b c}^{2}=N\left(N^{2}-1\right)=24 \quad C_{K}=\frac{N^{2}-1}{N}=\frac{8}{3}
$$

and for a single RH stop, we must use $2 n_{f}=1$. Then we get

$$
\begin{aligned}
\left|\mathcal{M}\left(g g \rightarrow \tilde{t}_{R} \tilde{t}_{R}\right)\right|^{2}= & 4 g_{3}^{4}\left[\frac{32}{3}+24 \frac{t-m^{2}}{s}+24 \frac{\left(t-m^{2}\right)^{2}}{s^{2}}+48 \frac{m^{2}}{s}\right. \\
& \left.+\frac{128}{3} \frac{m^{4}}{\left(t-m^{2}\right)^{2}}+\frac{128}{3} \frac{m^{2}}{t-m^{2}}\left(1+\frac{1}{4} \frac{m^{2}}{s}\right)\right]
\end{aligned}
$$


which coincide with our result above eq. (77).

Now integrate over $t$ and obtain

$$
\begin{aligned}
\sigma(m, s)= & 32 \frac{4 \pi \alpha_{3}^{2}}{s-4 m^{2}}\left[\sqrt{1-\frac{4 m^{2}}{s}}\left(\frac{5}{24}+\frac{31}{12} \frac{m^{2}}{s}\right)\right. \\
& \left.+\frac{4}{3} \frac{m^{2}}{s}\left(1+\frac{1}{4} \frac{m^{2}}{s}\right) \log \left(\frac{1-\sqrt{1-\frac{4 m^{2}}{s}}}{1+\sqrt{1-\frac{4 m^{2}}{s}}}\right)\right],
\end{aligned}
$$

which coincides with [26] allowing for the exchange of initial and final state $\left(s-4 m^{2} \rightarrow s\right.$ in the denominator) and the initial state averaging, i.e. a factor of $1 / 64$ for the two gluons initial state.

\section{B Annihilation into $S U(2)_{L}$ gauge bosons}

Another important channel of annihilation for light stops or staus is into EW gauge bosons. Let us consider first the pure $S U(2)_{L}$ case, neglecting the gauge boson masses, but with a split $S U(2)$ multiplet. We consider here the case of one left-handed sparticle and one left-handed antisparticle of momenta $p_{1}, p_{2}$, mass $m_{1}$ and $S U(2)$ index 1, annihilating into 2 gauge bosons of $S U(2)_{L}$ index $i, j$, momenta $p_{3}, p_{4}$ and Lorentz indices $\mu, \nu$ respectively. Then we can directly use the result for $S U(N)$, only taking into account that $T^{a} \rightarrow \sigma^{i} / 2$, with $\sigma^{i}$ denoting the Pauli matrices, and that in this case we have an initial state made

of the upper components of the $S U(2)_{L}$ doublet, while the lower component is exchanged in the t- and u-channel and can have a different mass $m_{2}$.

We have then for the two amplitudes, symmetric and antisymmetric in the group and Lorentz indices,

$$
\mathcal{A}_{s y m}^{\mu \nu}=i \frac{g_{2}^{2}}{8}\left\{\sigma^{i}, \sigma^{j}\right\}_{11}\left[\frac{\left(2 p_{1}-p_{3}\right)^{\mu}\left(2 p_{2}-p_{4}\right)^{\nu}}{t-m_{2}^{2}}+\frac{\left(2 p_{1}-p_{4}\right)^{\nu}\left(2 p_{2}-p_{3}\right)^{\mu}}{u-m_{2}^{2}}+2 g^{\mu \nu}\right]
$$

and

$$
\begin{aligned}
\mathcal{A}_{\text {asym }}^{\mu \nu}= & i \frac{g_{2}^{2}}{8}\left[\sigma^{i}, \sigma^{j}\right]_{11}\left[-\frac{\left(2 p_{1}-p_{3}\right)^{\mu}\left(2 p_{2}-p_{4}\right)^{\nu}}{t-m_{2}^{2}}+\frac{\left(2 p_{1}-p_{4}\right)^{\nu}\left(2 p_{2}-p_{3}\right)^{\mu}}{u-m_{2}^{2}}\right. \\
& \left.-2 \frac{g^{\mu \nu}(t-u)-\left(2 p_{4}+p_{3}\right)^{\mu}\left(p_{1}-p_{2}\right)^{\nu}+\left(p_{1}-p_{2}\right)^{\mu}\left(2 p_{3}+p_{4}\right)^{\nu}}{s}\right] .
\end{aligned}
$$

To compute the annihilation cross section, we have to sum over all the final states and initial states; this means that we have to sum over the $\mathrm{W}$ polarisations and over the $S U(2)_{L}$ indices $i, j$, but in this case the initial state group indices are fixed.

The symmetric piece gives

$$
\begin{aligned}
\left|\mathcal{A}_{\text {sym }}\right|^{2}= & \frac{g_{2}^{4}}{16}\left|\left\{\sigma^{i}, \sigma^{j}\right\}_{11}\right|^{2}\left[\frac{\left(t+m_{1}^{2}\right)^{2}}{\left(t-m_{2}^{2}\right)^{2}}+\frac{\left(u+m_{1}^{2}\right)^{2}}{\left(u-m_{2}^{2}\right)^{2}}+\frac{1}{2} \frac{\left(s-4 m_{1}^{2}\right)^{2}}{\left(t-m_{2}^{2}\right)\left(u-m_{2}^{2}\right)}\right. \\
& \left.+4+\frac{s / 2-4 m_{1}^{2}-2\left(t-m_{1}^{2}\right)}{t-m_{2}^{2}}+\frac{s / 2-4 m_{1}^{2}-2\left(u-m_{1}^{2}\right)}{u-m_{2}^{2}}\right]
\end{aligned}
$$




$$
\begin{aligned}
= & \frac{g_{2}^{4}}{4}\left|\left\{\sigma^{i}, \sigma^{j}\right\}_{11}\right|^{2}\left[\frac{1}{2}+\frac{1}{2} \frac{\left(m_{1}^{2}+m_{2}^{2}\right)^{2}}{\left(t-m_{2}^{2}\right)^{2}}\right. \\
& \left.+\frac{1}{t-m_{2}^{2}}\left(\frac{3 m_{2}^{2}+m_{1}^{2}}{2}-\frac{\left(m_{1}^{2}+m_{2}^{2}\right)^{2}}{s+2 m_{2}^{2}-2 m_{1}^{2}}\right)\right] .
\end{aligned}
$$

In the antisymmetric part instead gives

$$
\begin{aligned}
\left|\mathcal{A}_{\text {asym }}\right|^{2}= & \frac{g_{2}^{4}}{16}\left|\left[\sigma^{i}, \sigma^{j}\right]_{11}\right|^{2}\left[\frac{\left(t+m_{1}^{2}\right)^{2}}{\left(t-m_{2}^{2}\right)^{2}}+\frac{\left(u+m_{1}^{2}\right)^{2}}{\left(u-m_{2}^{2}\right)^{2}}-4\right. \\
& -\frac{1}{2} \frac{\left(s-4 m_{1}^{2}\right)^{2}}{\left(t-m_{2}^{2}\right)\left(u-m_{2}^{2}\right)}+2 \frac{(t-u)^{2}}{s^{2}}+\frac{16 m_{1}^{2}}{s} \\
& +\frac{(t-u)\left(3 / 2 s-t-3 m_{1}^{2}\right)+2\left(s-4 m_{1}^{2}\right)\left(u-m_{1}^{2}\right)}{s\left(t-m_{2}^{2}\right)} \\
& \left.+\frac{(u-t)\left(3 / 2 s-u-3 m_{1}^{2}\right)+2\left(s-4 m_{1}^{2}\right)\left(t-m_{1}^{2}\right)}{s\left(u-m_{2}^{2}\right)}\right] \\
= & \frac{g_{2}^{4}}{4}\left|\left[\sigma^{i}, \sigma^{j}\right]_{11}\right|^{2}\left[\frac{(t-u)^{2}}{2 s^{2}}+\frac{5 m_{1}^{2}-m_{2}^{2}}{s}+\frac{1}{2} \frac{\left(m_{1}^{2}+m_{2}^{2}\right)^{2}}{\left(t-m_{2}^{2}\right)^{2}}\right. \\
& \left.+\frac{1}{t-m_{2}^{2}}\left(\frac{m_{2}^{2}+3 m_{1}^{2}}{2}+\frac{\left(m_{2}^{2}+m_{1}^{2}\right)^{2}}{s+2 m_{2}^{2}-2 m_{1}^{2}}-\frac{\left(m_{2}^{2}-m_{1}^{2}\right)^{2}}{s}\right)\right] .
\end{aligned}
$$

\section{B.1 $S U(2)_{L}$ sum and total matrix element}

In this case the sum over the indices $i, j$ is simple. We have that

$$
\sum_{i, j} \frac{1}{4}\left|\left\{\sigma^{i}, \sigma^{j}\right\}_{11}\right|^{2}=\sum_{i, j} \frac{1}{4}\left|2 \delta_{i}^{j} I_{11}\right|^{2}=\sum_{i} \delta_{i}^{i}=2+1
$$

where we have considered the annihilation into $W^{1,2}$ separately from that into $W_{3}$. In fact the intermediate particle has a different mass in the two cases.

On the other hand the antisymmetric product gives

$$
\sum_{i, j} \frac{1}{4}\left|\left[\sigma^{i}, \sigma^{j}\right] 11\right|^{2}=\sum_{i, j} \frac{1}{4}\left|2 \epsilon_{i j k} \sigma_{11}^{k}\right|^{2}=\sum_{i, j}\left|\epsilon_{i j 3}\right|^{2}=2
$$

since in this case only $W^{3}$ can be exchanged in the s-channel for $W^{1,2}$ in the final state.

Then the matrix element for annihilation into $W^{1,2}$ gauge bosons is given by

$$
\begin{aligned}
\left|\mathcal{M}_{W 12}\right|^{2}= & g_{2}^{4}\left[1+\frac{(t-u)^{2}}{s^{2}}+\frac{10 m_{1}^{2}-2 m_{2}^{2}}{s}+2 \frac{\left(m_{1}^{2}+m_{2}^{2}\right)^{2}}{\left(t-m_{2}^{2}\right)^{2}}\right. \\
& \left.+\frac{4}{t-m_{2}^{2}}\left(m_{2}^{2}+m_{1}^{2}-\frac{\left(m_{2}^{2}-m_{1}^{2}\right)^{2}}{2 s}\right)\right]
\end{aligned}
$$

while the annihilation into $W^{3}$ has only the abelian contribution with the presence of a single mass $m_{1}$

$$
\left|\mathcal{M}_{W 3}\right|^{2}=g_{2}^{4}\left[\frac{1}{2}+\frac{2 m_{1}^{4}}{\left(t-m_{1}^{2}\right)^{2}}+\frac{2 m_{1}^{2}}{t-m_{1}^{2}}\left(1-\frac{2 m_{1}^{2}}{s}\right)\right] .
$$


The cross section for the first case is then

$$
\begin{aligned}
\sigma_{W 12}= & \frac{2 \pi \alpha_{2}^{2}}{s-4 m_{1}^{2}}\left[\sqrt{1-\frac{4 m_{1}^{2}}{s}}\left(\frac{2}{3}+\frac{13}{3} \frac{m_{1}^{2}}{s}-\frac{m_{2}^{2}}{s}+\frac{\left(m_{1}^{2}+m_{2}^{2}\right)^{2}}{s m_{2}^{2}+\left(m_{2}^{2}-m_{1}^{2}\right)^{2}}\right)\right. \\
& +2\left(\frac{m_{2}^{2}+m_{1}^{2}}{s}-\frac{\left(m_{2}^{2}-m_{1}^{2}\right)^{2}}{2 s^{2}}\right) \times \\
& \left.\times \log \left(\frac{s+2\left(m_{2}^{2}-m_{1}^{2}\right)-\sqrt{s\left(s-4 m_{1}^{2}\right)}}{s+2\left(m_{2}^{2}-m_{1}^{2}\right)+\sqrt{s\left(s-4 m_{1}^{2}\right)}}\right)\right],
\end{aligned}
$$

while the annihilation into $W^{3}$ is identical to the abelian one in eq. (9) for $e_{X}=1 / 2$.

\section{B.2 Annihilation into physical $W^{+} W^{-}$}

Let us now consider the case of a broken $S U(2)_{L}$ symmetry like the Standard Model and massive gauge bosons which mix to give the physical $W^{+}, W^{-}, Z, \gamma$. At the same time let us consider a general initial state given by the light stau mass eigenstate $\tilde{\tau}_{1}=$ $\tilde{\tau}_{L} \cos \theta_{\tilde{\tau}}+\tilde{\tau}_{R} \sin \theta_{\tilde{\tau}}$ and its antiparticle. In this case the intermediate particle exchanged in the t- and u-channel can be only a left-handed sneutrino and therefore we can neglect the mixing for the intermediate state.

Then the annihilation into $W^{+} W^{-}$is given by the following channels:

t sneutrino exchange in the t-channel described by the amplitude

$$
\mathcal{A}_{t}^{\mu \nu}=i \frac{g_{2}^{2}}{2} \cos ^{2} \theta_{\tilde{\tau}} \frac{\left(2 p_{1}-p_{3}\right)^{\mu}\left(2 p_{2}-p_{4}\right)^{\nu}}{t-m_{\tilde{\nu}}^{2}} ;
$$

u NO u-channel since $W^{+}$and $W^{-}$are different particles !

4 supersymmetric four-scalar coupling giving the amplitude:

$$
\mathcal{A}_{4}^{\mu \nu}=i \frac{g_{2}^{2}}{2} \cos ^{2} \theta_{\tilde{\tau}} g^{\mu \nu} ;
$$

this contribution is symmetric in the exchange of $\mu, \nu$;

s off-shell $Z / \gamma$ in the s-channel decaying into two $W W$ via the non-abelian interaction:

$$
\begin{aligned}
\mathcal{A}_{s}^{\mu \nu}= & i \frac{g_{2}^{2}}{2} \cos ^{2} \theta_{\tilde{\tau}}\left(1-\frac{4}{3} \frac{\sin ^{2} \theta_{W}}{\cos ^{2} \theta_{\tilde{t}}}\right) \frac{1}{s-M_{Z}^{2}}\left[g^{\mu \nu}(t-u)\right. \\
& \left.-\left(2 p_{4}+p_{3}\right)^{\mu}\left(p_{1}-p_{2}\right)^{\nu}+\left(p_{1}-p_{2}\right)^{\mu}\left(2 p_{3}+p_{4}\right)^{\nu}\right] \\
& +i e^{2} \frac{2}{3} \frac{1}{s}\left[g^{\mu \nu}(t-u)-\left(2 p_{4}+p_{3}\right)^{\mu}\left(p_{1}-p_{2}\right)^{\nu}\right. \\
& \left.+\left(p_{1}-p_{2}\right)^{\mu}\left(2 p_{3}+p_{4}\right)^{\nu}\right] \\
= & i \frac{g_{2}^{2}}{2} \cos ^{2} \theta_{\tilde{\tau}}\left(1-\frac{4}{3} \frac{\sin ^{2} \theta_{W}}{\cos ^{2} \theta_{\tilde{\tau}}} \frac{M_{Z}^{2}}{s}\right) \\
& \frac{g^{\mu \nu}(t-u)-\left(2 p_{4}+p_{3}\right)^{\mu}\left(p_{1}-p_{2}\right)^{\nu}+\left(p_{1}-p_{2}\right)^{\mu}\left(2 p_{3}+p_{4}\right)^{\nu}}{s-M_{Z}^{2}} ;
\end{aligned}
$$


this contribution is completely antisymmetric under the exchange of the $\mathrm{W}$ momenta and Lorentz indices. Note that the photon contribution is proportional to $e^{2}=$ $g_{2}^{2} \sin ^{2} \theta_{W}$ and cancels exactly with the second term due to the Z-boson in the case of equal mass. In that limit in fact the $U(1)_{Y}$ factor decouples and does not participate in the non-abelian interaction.

s-H off-shell $h / H$ in the s-channel decaying into two $W W$ via the non-abelian interaction; in this case we have to consider both neutral Higgses:

$$
\mathcal{A}_{s H}^{\mu \nu}=i \frac{g_{2}^{2}}{2} \cos ^{2} \theta_{\tilde{\tau}} g^{\mu \nu} \frac{M_{W}^{2}}{s}\left[\frac{C_{H} s}{s-M_{H}^{2}}+\frac{C_{h} s}{s-M_{h}^{2}}\right] ;
$$

where $C_{H / h}$ is coming from the product of the coupling of the staus to the Higgses and of the Higgses to the WW pair. These constants depend on the whole SUSY breaking parameters. For the staus these couplings are probably negligible. We have in fact

$$
\begin{aligned}
C_{H / h}= & \frac{\left(Z_{1 H / h}\right)^{2}-\left(Z_{2 H / h}\right)^{2} \tan ^{2} \beta}{\left(1+\tan ^{2} \beta\right) \cos ^{4} \theta_{W}}\left(1-\frac{4}{3} \sin ^{2} \theta_{W}\left(1-\tan ^{2} \theta_{\tilde{\tau}}\right)\right) \\
& +4 \frac{Y_{\tau}^{2} \tan \beta Z_{2 H / h}\left(Z_{1 H / h}+Z_{2 H / h} \tan \beta\right)}{g_{2}^{2} \cos ^{2} \theta_{W}\left(1+\tan ^{2} \beta\right)}\left(1+\tan ^{2} \theta_{\tilde{\tau}}\right) \\
& -\tan \theta_{\tilde{\tau}} \frac{\sqrt{2}\left(Z_{1 H / h}+Z_{2 H / h} \tan \beta\right)}{g_{2} \cos ^{2} \theta_{W} M_{W} \sqrt{1+\tan ^{2} \beta}} \times \\
& \times\left(Z_{2 H / h} A_{\tau}+Z_{1 H / h}\left(A_{\tau}^{\prime}+\mu^{\star} Y_{\tau}\right)+\text { h.c. }\right),
\end{aligned}
$$

where $Z$ is the matrix which diagonalises the Higgs mass matrix, $Y_{\tau}$ is the tau Yukawa coupling, $A_{\tau}, A_{\tau}^{\prime}$ are the SUSY breaking trilinear terms and $\mu$ the Higgs supersymmetric mass parameter. This contribution is suppressed by $M_{W}^{2} / s$ for large $s$. We can include it easily into the 4-vertex contribution by substituting

$$
1 \rightarrow 1+\frac{C_{H} M_{W}^{2}}{s-M_{H}^{2}}+\frac{C_{h} M_{W}^{2}}{s-M_{h}^{2}}=1+K_{H}(s) .
$$

Now we can write the t-channel as the sum of a symmetric and antisymmetric part, adding and subtracting a fictitious u-channel, as

$$
\begin{aligned}
\frac{\left(2 p_{1}-p_{3}\right)^{\mu}\left(2 p_{2}-p_{4}\right)^{\nu}}{t-m_{\tilde{\nu}}^{2}} & =\frac{1}{2}\left[\frac{\left(2 p_{1}-p_{3}\right)^{\mu}\left(2 p_{2}-p_{4}\right)^{\nu}}{t-m_{\tilde{\nu}}^{2}}+\frac{\left(2 p_{1}-p_{4}\right)^{\nu}\left(2 p_{2}-p_{3}\right)^{\mu}}{u-m_{\tilde{\nu}}^{2}}\right] \\
& +\frac{1}{2}\left[\frac{\left(2 p_{1}-p_{3}\right)^{\mu}\left(2 p_{2}-p_{4}\right)^{\nu}}{t-m_{\tilde{\nu}}^{2}}-\frac{\left(2 p_{1}-p_{4}\right)^{\nu}\left(2 p_{2}-p_{3}\right)^{\mu}}{u-m_{\tilde{\nu}}^{2}}\right]
\end{aligned}
$$

so that we can make contact with the previous computation and find for the symmetric and antisymmetric amplitudes respectively:

$$
\begin{aligned}
\mathcal{A}_{\text {sym }}^{\mu \nu}= & +i \frac{g_{2}^{2}}{4} \cos ^{2} \theta_{\tilde{\tau}}\left[\frac{\left(2 p_{1}-p_{3}\right)^{\mu}\left(2 p_{2}-p_{4}\right)^{\nu}}{t-m_{\tilde{\nu}}^{2}}\right. \\
& \left.+\frac{\left(2 p_{1}-p_{4}\right)^{\nu}\left(2 p_{2}-p_{3}\right)^{\mu}}{u-m_{\tilde{\nu}}^{2}}+2 g^{\mu \nu}\left(1+K_{H}(s)\right)\right]
\end{aligned}
$$


and

$$
\begin{aligned}
\mathcal{A}_{\text {asym }}^{\mu \nu}= & i \frac{g_{2}^{2}}{4} \cos ^{2} \theta_{\tilde{\tau}}\left[\frac{\left(2 p_{1}-p_{3}\right)^{\mu}\left(2 p_{2}-p_{4}\right)^{\nu}}{t-m_{\tilde{\nu}}^{2}}\right. \\
& -\frac{\left(2 p_{1}-p_{4}\right)^{\nu}\left(2 p_{2}-p_{3}\right)^{\mu}}{u-m_{\tilde{\nu}}^{2}}+2\left(1-G_{Z}(s)\right) \times \\
& \left.\times \frac{g^{\mu \nu}(t-u)-\left(2 p_{4}+p_{3}\right)^{\mu}\left(p_{1}-p_{2}\right)^{\nu}+\left(p_{1}-p_{2}\right)^{\mu}\left(2 p_{3}+p_{4}\right)^{\nu}}{s-M_{Z}^{2}}\right] .
\end{aligned}
$$

where $G_{Z}(s)=\frac{4}{3} \frac{\sin ^{2} \theta_{W}}{\cos ^{2} \theta_{\tilde{\tau}}} \frac{M_{Z}^{2}}{s}$ vanishes in the limit of zero Z mass. This coincides with the previous result for $K_{H}, G_{Z}, M_{Z}=0$, a part for a sign, which just corresponds in exchanging $i \leftrightarrow j$.

\section{B.3 Polarisation sum}

The sum over the $\mathrm{W}$ polarisation in this case is given by the polarisation tensor

$$
\Pi^{\mu \mu^{\prime}}=-g^{\mu \mu^{\prime}}+\frac{p_{3}^{\mu} p_{3}^{\mu^{\prime}}}{M_{W}^{2}}
$$

where $p_{3}$ is the gauge boson momentum.

We have then for the matrix element

$$
|\mathcal{M}|^{2}=\mathcal{A}_{\mu \nu}^{*} \mathcal{A}^{\mu \nu}-\frac{\left|p_{3}^{\mu} A_{\mu \nu}\right|^{2}}{M_{W}^{2}}-\frac{\left|p_{4}^{\nu} A_{\mu \nu}\right|^{2}}{M_{W}^{2}}+\frac{\left|p_{3}^{\mu} p_{4}^{\nu} A_{\mu \nu}\right|^{2}}{M_{W}^{4}}
$$

in this case neither amplitude vanishes when contracted with the gauge boson's momentum. Note that the second and third contributions are related again by the symmetry $p_{3} \leftrightarrow p_{4} ; \nu \leftrightarrow \mu$ and are equal since the final state has two particle with the same mass.

\section{B.4 Symmetric part}

We must compute the four contributions, and we have then

$$
\begin{aligned}
\mathcal{A}_{\mu \nu}^{*} \mathcal{A}^{\mu \nu}= & \frac{g_{2}^{4} \cos ^{4} \theta_{\tilde{\tau}}}{2}\left[1+\frac{\left(m_{\tilde{\nu}}^{2}+m_{\tilde{\tau}}^{2}-M_{W}^{2} / 2\right)^{2}}{\left(t-m_{\tilde{\nu}}^{2}\right)^{2}}+2 K_{H}(s)\left(1+K_{H}(s)\right)\right. \\
& +\frac{1}{2} \frac{1}{t-m_{\tilde{\nu}}^{2}}\left(4\left(m_{\tilde{\nu}}^{2}+m_{\tilde{\tau}}^{2}\right)-2 M_{W}^{2}+s-4\left(m_{\tilde{\nu}}^{2}+m_{\tilde{\tau}}^{2}\right)+2 M_{W}^{2}\right. \\
& \left.\left.-\frac{\left(s-4 m_{\tilde{\tau}}^{2}+M_{W}^{2}\right)^{2}}{s+2\left(m_{\tilde{\nu}}^{2}-m_{\tilde{\tau}}^{2}-M_{W}^{2}\right)}+K_{H}(s)\left(s-4 m_{\tilde{\nu}}^{2}-4 m_{\tilde{\tau}}^{2}+2 M_{W}^{2}\right)\right)\right] \\
= & \frac{g_{2}^{4} \cos ^{4} \theta_{\tilde{\tau}}}{2}\left[1+\frac{\left(m_{\tilde{\nu}}^{2}+m_{\tilde{\tau}}^{2}-M_{W}^{2} / 2\right)^{2}}{\left(t-m_{\tilde{\nu}}^{2}\right)^{2}}+2 K_{H}(s)\left(1+K_{H}(s)\right)\right. \\
& +\frac{1}{t-m_{\tilde{\nu}}^{2}}\left(m_{\tilde{\nu}}^{2}+3 m_{\tilde{\tau}}^{2}-2 M_{W}^{2}-\frac{1}{2} \frac{\left(2 m_{\tilde{\tau}}^{2}+2 m_{\tilde{\nu}}^{2}-3 M_{W}^{2}\right)^{2}}{s\left(m_{\tilde{\nu}}^{2}-m_{\tilde{\tau}}^{2}-M_{W}^{2}\right)}\right. \\
& \left.\left.+\frac{K_{H}(s)}{2}\left(s-4 m_{\tilde{\nu}}^{2}-4 m_{\tilde{\tau}}^{2}+2 M_{W}^{2}\right)\right)\right]
\end{aligned}
$$

which in the limit of vanishing $M_{W}$ and $m_{\tilde{\nu}}=m_{\tilde{\tau}}$ coincides with our old result. 
The other pieces give instead

$$
\begin{aligned}
\frac{\left|p_{3}^{\mu} A_{\mu \nu}\right|^{2}}{M_{W}^{2}}+\frac{\left|p_{4}^{\nu} A_{\mu \nu}\right|^{2}}{M_{W}^{2}}= & \frac{g_{2}^{4} \cos ^{4} \theta_{\tilde{\tau}}}{4}\left[\frac { ( m _ { \tilde { \nu } } ^ { 2 } - m _ { \tilde { \tau } } ^ { 2 } ) ^ { 2 } } { M _ { W } ^ { 2 } } \left(\frac{2 m_{\tilde{\nu}}^{2}+2 m_{\tilde{\tau}}^{2}-M_{W}^{2}}{\left(t-m_{\tilde{\nu}}^{2}\right)^{2}}\right.\right. \\
& \left.+\frac{2}{t-m_{\tilde{\nu}}^{2}} \frac{\left.2 m_{\tilde{\tilde{\nu}}}^{2}+2 m_{\tilde{\tau}}^{2}-3 M_{W}^{2}\right)^{2}}{s+2\left(m_{\tilde{\nu}}^{2}-m_{\tilde{\tau}}^{2}-M_{W}^{2}\right)}\right) \\
& \left.-4 K_{H}(s) \frac{m_{\tilde{\nu}}^{2}-m_{\tilde{\tau}}^{2}}{M_{W}^{2}}\left(1+\frac{m_{\tilde{\nu}}^{2}-m_{\tilde{\tau}}^{2}+s / 2}{t-m_{\tilde{\nu}}^{2}}\right)+2 K_{H}^{2}(s)\right]
\end{aligned}
$$

and the last part:

$$
\begin{aligned}
\frac{\left|p_{3}^{\mu} p_{4}^{\nu} A_{\mu \nu}\right|^{2}}{M_{W}^{4}}= & \frac{g_{2}^{4} \cos ^{4} \theta_{\tilde{\tau}}}{4}\left[\frac { ( m _ { \tilde { \nu } } ^ { 2 } - m _ { \tilde { \tau } } ^ { 2 } ) ^ { 2 } } { M _ { W } ^ { 4 } } \left(1+\frac{1}{2} \frac{\left(m_{\tilde{\nu}}^{2}-m_{\tilde{\tau}}^{2}\right)^{2}}{\left(t-m_{\tilde{\nu}}^{2}\right)^{2}}\right.\right. \\
& \left.+2 \frac{m_{\tilde{\nu}}^{2}-m_{\tilde{\tau}}^{2}}{t-m_{\tilde{\nu}}^{2}}\left(1-\frac{1}{4} \frac{m_{\tilde{\nu}}^{2}-m_{\tilde{\tau}}^{2}}{s+2\left(m_{\tilde{\nu}}^{2}-m_{\tilde{\tau}}^{2}+M_{W}^{2}\right)}\right)\right) \\
& +K_{H}(s) \frac{m_{\tilde{\nu}}^{2}-m_{\tilde{\tau}}^{2}}{M_{W}^{2}}\left(\frac{s}{M_{W}^{2}}-2\right)\left(1+\frac{m_{\tilde{\nu}}^{2}-m_{\tilde{\tau}}^{2}}{t-m_{\tilde{\nu}}^{2}}\right) \\
& \left.+\frac{1}{4} K_{H}^{2}(s)\left(\frac{s}{M_{W}^{2}}-2\right)^{2}\right] .
\end{aligned}
$$

Both these contributions vanish in the limit of equal stau and sneutrino masses and zero gauge boson mass as they should.

So summing all together the result is

$$
\begin{aligned}
\left|\mathcal{M}_{\text {sym }}\right|^{2}= & \frac{g_{2}^{4} \cos ^{4} \theta_{\tilde{\tau}}}{2}\left[1+\frac{\left(m_{\tilde{\nu}}^{2}+m_{\tilde{\tau}}^{2}-M_{W}^{2} / 2\right)^{2}}{\left(t-m_{\tilde{\nu}}^{2}\right)^{2}}\right. \\
& +\frac{1}{t-m_{\tilde{\nu}}^{2}}\left(m_{\tilde{\nu}}^{2}+3 m_{\tilde{\tau}}^{2}-2 M_{W}^{2}-\frac{1}{2} \frac{\left(2 m_{\tilde{\tau}}^{2}+2 m_{\tilde{\nu}}^{2}-3 M_{W}^{2}\right)^{2}}{s+2\left(m_{\tilde{\nu}}^{2}-m_{\tilde{\tau}}^{2}-M_{W}^{2}\right)}\right) \\
& -\frac{\left(m_{\tilde{\nu}}^{2}-m_{\tilde{\tau}}^{2}\right)^{2}}{M_{W}^{2}\left(t-m_{\tilde{\nu}}^{2}\right)}\left(\frac{m_{\tilde{\nu}}^{2}+m_{\tilde{\tau}}^{2}-M_{W}^{2} / 2}{t-m_{\tilde{\nu}}^{2}}+\frac{2 m_{\tilde{\tau}}^{2}+2 m_{\tilde{\nu}}^{2}-3 M_{W}^{2}}{s+2\left(m_{\tilde{\nu}}^{2}-m_{\tilde{\tau}}^{2}-M_{W}^{2}\right)}\right) \\
& +\frac{1}{2} \frac{\left(m_{\tilde{\nu}}^{2}-m_{\tilde{\tau}}^{2}\right)^{2}}{M_{W}^{4}}\left(1+\frac{1}{2} \frac{\left(m_{\tilde{\nu}}^{2}-m_{\tilde{\tau}}^{2}\right)^{2}}{\left(t-m_{\tilde{\nu}}^{2}\right)^{2}}\right. \\
& \left.+2 \frac{m_{\tilde{\nu}}^{2}-m_{\tilde{\tau}}^{2}}{t-m_{\tilde{\nu}}^{2}}\left(1-\frac{1}{4} \frac{m_{\tilde{\nu}}^{2}-m_{\tilde{\tau}}^{2}}{s+2\left(m_{\tilde{\nu}}^{2}-m_{\tilde{\tau}}^{2}+M_{W}^{2}\right)}\right)\right) \\
& +K_{H}(s)\left(2-3 \frac{m_{\tilde{\nu}}^{2}-m_{\tilde{\tau}}^{2}}{M_{W}^{2}}+\frac{s}{2 M_{W}^{2}} \frac{m_{\tilde{\nu}}^{2}-m_{\tilde{\tau}}^{2}}{M_{W}^{2}}\right) \\
& +\frac{K_{H}(s)}{t-m_{\tilde{\nu}}^{2}}\left(\frac{s}{2}-2 m_{\tilde{\nu}}^{2}-2 m_{\tilde{\tau}}^{2}+M_{W}^{2}\right. \\
& \left.-\frac{m_{\tilde{\nu}}^{2}-m_{\tilde{\tau}}^{2}}{M_{W}^{2}}\left(s+3\left(m_{\tilde{\nu}}^{2}-m_{\tilde{\tau}}^{2}\right)\left(1-\frac{s}{2 M_{W}^{2}}\right)\right)\right) \\
& \left.+K_{H}^{2}(s)\left(\frac{7}{2}-\frac{s}{2 M_{W}^{2}}+\frac{s^{2}}{8 M_{W}^{4}}\right)\right] .
\end{aligned}
$$

Note that the in the limit of large $s, s K_{H}(s)$ remains finite and therefore there is no problem with unitarity. 


\section{B.5 Antisymmetric part}

The antisymmetric piece is more involved. We have

$$
\begin{aligned}
\mathcal{A}_{\mu \nu}^{*} \mathcal{A}^{\mu \nu}= & \frac{g_{2}^{4} \cos ^{4} \theta_{\tilde{\tau}}}{4}\left[2+2 \frac{\left(m_{\tilde{\nu}}^{2}+m_{\tilde{\tau}}^{2}-M_{W}^{2} / 2\right)^{2}}{\left(t-m_{\tilde{\nu}}^{2}\right)^{2}}\right. \\
& +\frac{1}{t-m_{\tilde{\nu}}^{2}}\left(4\left(m_{\tilde{\nu}}^{2}+m_{\tilde{\tau}}^{2}\right)-2 M_{W}^{2}+\frac{\left(s-4 m_{\tilde{\tau}}^{2}+M_{W}^{2}\right)^{2}}{s+2\left(m_{\tilde{\nu}}^{2}-m_{\tilde{\tau}}^{2}-M_{W}^{2}\right)}\right) \\
& +\left(1-G_{Z}(s)\right)^{2} \frac{5 / 2(t-u)^{2}-4\left(s-4 m_{\tilde{\tau}}^{2}\right)\left(s+M_{W}^{2} / 2\right)}{\left(s-M_{Z}^{2}\right)^{2}} \\
& -\left(1-G_{Z}(s)\right) \frac{2(t-u)+4\left(s-4 m_{\tilde{\tau}}^{2}\right)}{s-M_{Z}^{2}} \\
& +\left(1-G_{Z}(s)\right) \frac{(t-u)\left(3 s-2 m_{\tilde{\nu}}^{2}-6 m_{\tilde{\tau}}^{2}+2 M_{W}^{2}\right)}{\left(s-M_{Z}^{2}\right)\left(t-m_{\tilde{\nu}}^{2}\right)} \\
& \left.-\left(1-G_{Z}(s)\right) \frac{4\left(s+m_{\tilde{\nu}}^{2}-m_{\tilde{\tau}}^{2}\right)\left(s-4 m_{\tilde{\tau}}^{2}\right)}{\left(s-M_{Z}^{2}\right)\left(t-m_{\tilde{\nu}}^{2}\right)}\right]
\end{aligned}
$$

which in the limit of vanishing $M_{W}, M_{Z}$ and $m_{\tilde{\nu}}=m_{\tilde{\tau}}$ coincides with our old result.

The other pieces give instead

$$
\begin{aligned}
& \frac{\left|p_{3}^{\mu} A_{\mu \nu}\right|^{2}}{M_{W}^{2}}+\frac{\left|p_{4}^{\nu} A_{\mu \nu}\right|^{2}}{M_{W}^{2}}=\frac{g_{2}^{4} \cos ^{4} \theta_{\tilde{\tau}}}{2} \times \\
\times & {\left[\frac{1}{2} \frac{(t-u)^{2}}{\left(s-M_{Z}^{2}\right)^{2}}\left(1-G_{Z}(s)\right)\left(\frac{M_{Z}^{2}}{M_{W}^{2}}-\frac{1}{2}-G_{Z}(s)\left(\frac{s}{M_{W}^{2}}-\frac{1}{2}\right)\right)\right.} \\
& -\frac{\left(s-4 m_{\tilde{\tau}}^{2}\right) M_{W}^{2}}{\left(s-M_{Z}^{2}\right)^{2}}\left(\frac{M_{Z}^{2}}{M_{W}^{2}}-1-G_{Z}(s)\left(\frac{s}{M_{W}^{2}}-1\right)\right)^{2} \\
& \left.-\frac{m_{\tilde{\nu}}^{2}-m_{\tilde{\tau}}^{2} t-u-2\left(s-4 m_{\tilde{\tau}}^{2}\right)}{s-M_{Z}^{2}} \frac{M_{Z}^{2}}{M_{W}^{2}}-1-G_{Z}(s)\left(\frac{s}{M_{W}^{2}}-1\right)\right) \\
& -\frac{\left(m_{\tilde{\nu}}^{2}-m_{\tilde{\tau}}^{2}\right)^{2}}{M_{W}^{2}\left(s-M_{Z}^{2}\right)}\left(1-G_{Z}(s)\right) \frac{t-u}{t-m_{\tilde{\nu}}^{2}} \\
& \left.+\frac{\left(m_{\tilde{\nu}}^{2}-m_{\tilde{\tau}}^{2}\right)^{2}}{M_{W}^{2}\left(t-m_{\tilde{\nu}}^{2}\right)}\left(1+\frac{s-4 m_{\tilde{\tau}}^{2}+M_{W}^{2}}{s+2\left(m_{\tilde{\nu}}^{2}-m_{\tilde{\tau}}^{2}-M_{W}^{2}\right)}+\frac{m_{\tilde{\nu}}^{2}+m_{\tilde{\tau}}^{2}-M_{W} / 2}{t-m_{\tilde{\nu}}^{2}}\right)\right] ;
\end{aligned}
$$

in the limit of vanishing $m_{\tilde{\nu}}^{2}-m_{\tilde{\tau}}^{2}, M_{Z}, M_{W}$ masses keeping $M_{Z} / M_{W} \rightarrow 1$ we have

$$
\frac{\left|p_{3}^{\mu} A_{\mu \nu}\right|^{2}}{M_{W}^{2}}+\frac{\left|p_{4}^{\nu} A_{\mu \nu}\right|^{2}}{M_{W}^{2}} \rightarrow \frac{g_{2}^{4} \cos ^{4} \theta_{\tilde{\tau}}}{4} \frac{(t-u)^{2}}{2 s},
$$

as expected from the QCD result.

The last part gives instead

$$
\begin{aligned}
\frac{\left|p_{3}^{\mu} p_{4}^{\nu} A_{\mu \nu}\right|^{2}}{M_{W}^{4}}= & \frac{g_{2}^{4} \cos ^{4} \theta_{\tilde{\tau}}}{16 M_{W}^{4}}\left[\frac{(t-u)^{2}}{\left(s-M_{Z}^{2}\right)^{2}}\left(M_{Z}^{2}-G_{Z}(s) s\right)^{2}\right. \\
& -4\left(m_{\tilde{\nu}}^{2}-m_{\tilde{\tau}}^{2}\right)^{2} \frac{t-u}{\left(t-m_{\tilde{\nu}}^{2}\right)\left(s-M_{Z}^{2}\right)}\left(M_{Z}^{2}-G_{Z}(s) s\right) \\
& \left.+2 \frac{\left(m_{\tilde{\nu}}^{2}-m_{\tilde{\tau}}^{2}\right)^{4}}{\left(t-m_{\tilde{\nu}}^{2}\right)}\left(\frac{1}{\left(t-m_{\tilde{\nu}}^{2}\right)}+2 \frac{1}{s+2\left(m_{\tilde{\nu}}^{2}-m_{\tilde{\tau}}^{2}-M_{W}^{2}\right)}\right)\right] .
\end{aligned}
$$


Note that this contribution does not vanish in the limit of equal stop and sbottom masses and massless gauge bosons.In fact keeping $M_{Z} / M_{W} \rightarrow 1$, we have

$$
\frac{\left|p_{3}^{\mu} p_{4}^{\nu} A_{\mu \nu}\right|^{2}}{M_{W}^{4}} \rightarrow \frac{g_{2}^{4} \cos ^{4} \theta_{\tilde{\tau}}}{8} \frac{(t-u)^{2}}{2 s}
$$

which gives the annihilation into the Goldstone part of the Higgs field.

We can now put all together to give

$$
\begin{aligned}
& \left|\mathcal{M}_{\text {asym }}\right|^{2}=\frac{g_{2}^{4} \cos ^{4} \theta_{\tilde{\tau}}}{2}\left[1+\frac{\left(m_{\tilde{\nu}}^{2}+m_{\tilde{\tau}}^{2}-M_{W}^{2} / 2\right)^{2}}{\left(t-m_{\tilde{\nu}}^{2}\right)^{2}}\right. \\
& +\frac{1}{t-m_{\tilde{\nu}}^{2}}\left(2\left(m_{\tilde{\nu}}^{2}+m_{\tilde{\tau}}^{2}\right)-M_{W}^{2}+\frac{1}{2} \frac{\left(s-4 m_{\tilde{\tau}}^{2}+M_{W}^{2}\right)^{2}}{s+2\left(m_{\tilde{\nu}}^{2}-m_{\tilde{\tau}}^{2}-M_{W}^{2}\right)}\right) \\
& +\frac{1}{2}\left(1-G_{Z}(s)\right)^{2} \frac{5 / 2(t-u)^{2}-4\left(s-4 m_{\tilde{\tau}}^{2}\right)\left(s+M_{W}^{2} / 2\right)}{\left(s-M_{Z}^{2}\right)^{2}} \\
& -\left(1-G_{Z}(s)\right) \frac{(t-u)+2\left(s-4 m_{\tilde{\tau}}^{2}\right)}{s-M_{Z}^{2}} \\
& +\frac{1}{2}\left(1-G_{Z}(s)\right) \frac{(t-u)\left(3 s-2 m_{\tilde{\nu}}^{2}-6 m_{\tilde{\tau}}^{2}+2 M_{W}^{2}\right)}{\left(s-M_{Z}^{2}\right)\left(t-m_{\tilde{\nu}}^{2}\right)} \\
& -\left(1-G_{Z}(s)\right) \frac{2\left(s+m_{\tilde{\nu}}^{2}-m_{\tilde{\tau}}^{2}\right)\left(s-4 m_{\tilde{\tau}}^{2}\right)}{\left(s-M_{Z}^{2}\right)\left(t-m_{\tilde{\nu}}^{2}\right)} \\
& -\frac{(t-u)^{2}}{2\left(s-M_{Z}^{2}\right)^{2}}\left(1-G_{Z}(s)\right)\left(\frac{M_{Z}^{2}}{M_{W}^{2}}-\frac{1}{2}-G_{Z}(s)\left(\frac{s}{M_{W}^{2}}-\frac{1}{2}\right)\right) \\
& +\frac{M_{W}^{2}\left(s-4 m_{\tilde{\tau}}^{2}\right)}{\left(s-M_{Z}^{2}\right)^{2}}\left(\frac{M_{Z}^{2}}{M_{W}^{2}}-1-G_{Z}(s)\left(\frac{s}{M_{W}^{2}}-1\right)\right)^{2} \\
& +\frac{m_{\tilde{\nu}}^{2}-m_{\tilde{\tau}}^{2}}{s-M_{Z}^{2}}\left(\frac{M_{Z}^{2}}{M_{W}^{2}}-1-G_{Z}(s)\left(\frac{s}{M_{W}^{2}}-1\right)\right) \frac{t-u-2\left(s-4 m_{\tilde{\tau}}^{2}\right)}{t-m_{\tilde{\nu}}^{2}} \\
& +\frac{\left(m_{\tilde{\nu}}^{2}-m_{\tilde{\tau}}^{2}\right)^{2}}{M_{W}^{2}\left(s-M_{Z}^{2}\right)}\left(1-G_{Z}(s)\right) \frac{t-u}{t-m_{\tilde{\nu}}^{2}} \\
& -\frac{\left(m_{\tilde{\nu}}^{2}-m_{\tilde{\tau}}^{2}\right)^{2}}{M_{W}^{2}\left(t-m_{\tilde{\nu}}^{2}\right)^{2}}\left(m_{\tilde{\nu}}^{2}+m_{\tilde{\tau}}^{2}-M_{W} / 2\right) \\
& -\frac{\left(m_{\tilde{\nu}}^{2}-m_{\tilde{\tau}}^{2}\right)^{2}}{M_{W}^{2}\left(t-m_{\tilde{\nu}}^{2}\right)}\left(1+\frac{s-4 m_{\tilde{\tau}}^{2}+M_{W}^{2}}{s+2\left(m_{\tilde{\nu}}^{2}-m_{\tilde{\tau}}^{2}-M_{W}^{2}\right)}\right) \\
& +\frac{(t-u)^{2}}{8\left(s-M_{Z}^{2}\right)^{2}}\left(\frac{M_{Z}^{2}}{M_{W}^{2}}-G_{Z}(s) \frac{s}{M_{W}^{2}}\right)^{2} \\
& -\frac{1}{2} \frac{\left(m_{\tilde{\nu}}^{2}-m_{\tilde{\tau}}^{2}\right)^{2}}{M_{W}^{2}} \frac{t-u}{\left(t-m_{\tilde{\nu}}^{2}\right)\left(s-M_{Z}^{2}\right)}\left(\frac{M_{Z}^{2}}{M_{W}^{2}}-G_{Z}(s) \frac{s}{M_{W}^{2}}\right) \\
& \left.+\frac{1}{4} \frac{\left(m_{\tilde{\nu}}^{2}-m_{\tilde{\tau}}^{2}\right)^{4}}{M_{W}^{4}\left(t-m_{\tilde{\nu}}^{2}\right)}\left(\frac{1}{\left(t-m_{\tilde{\nu}}^{2}\right)}+2 \frac{1}{s+2\left(m_{\tilde{\nu}}^{2}-m_{\tilde{\tau}}^{2}-M_{W}^{2}\right)}\right)\right] .
\end{aligned}
$$

Note that to reduce these expressions in terms of only the $t$ variable, we have used the simple decompositions, i.e. from $s+t+u=2 m_{\tilde{t}}^{2}+2 M_{W}^{2}$ one obtains

$$
\frac{1}{\left(t-m_{\tilde{\nu}}^{2}\right)\left(u-m_{\tilde{\nu}}^{2}\right)}=-\frac{1}{s+2\left(m_{\tilde{\nu}}^{2}-m_{\tilde{\tau}}^{2}-M_{W}^{2}\right)}\left(\frac{1}{t-m_{\tilde{\nu}}^{2}}+\frac{1}{u-m_{\tilde{\nu}}^{2}}\right) .
$$




\section{B.6 Results for the cross section}

We can integrate the matrix element to obtain the cross section in the two cases:

$$
\begin{aligned}
\sigma_{s y m}(s)= & \frac{g_{2}^{4} \cos ^{4} \theta_{\tilde{\tau}}}{32 \pi\left(s-4 m_{\tilde{\tau}}^{2}\right)} \sqrt{\left(1-\frac{4 m_{\tilde{\tau}}^{2}}{s}\right)\left(1-\frac{4 M_{W}^{2}}{s}\right)}\left[1+\frac{1}{2} \frac{\left(m_{\tilde{\nu}}^{2}-m_{\tilde{\tau}}^{2}\right)^{2}}{M_{W}^{4}}\right. \\
& +\frac{\left(m_{\tilde{\nu}}^{2}+m_{\tilde{\tau}}^{2}-M_{W}^{2} / 2\right)^{2}}{m_{\tilde{\nu}}^{2}\left(s+m_{\tilde{\nu}}^{2}-2 m_{\tilde{\tau}}^{2}-2 M_{W}^{2}\right)+\left(m_{\tilde{\tau}}^{2}-M_{W}^{2}\right)^{2}} \times \\
& \times\left(1-\frac{\left(m_{\tilde{\nu}}^{2}-m_{\tilde{\tau}}^{2}\right)^{2}}{M_{W}^{2}\left(2 m_{\tilde{\nu}}^{2}+2 m_{\tilde{\tau}}^{2}-M_{W}^{2}\right)}\right)^{2} \\
& +K_{H}(s)\left(2-3 \frac{m_{\tilde{\nu}}^{2}-m_{\tilde{\tau}}^{2}}{M_{W}^{2}}+\frac{s}{2 M_{W}^{2}} \frac{m_{\tilde{\nu}}^{2}-m_{\tilde{\tau}}^{2}}{M_{W}^{2}}\right) \\
& +K_{H}^{2}(s)\left(\frac{7}{2}-\frac{s}{2 M_{W}^{2}}+\frac{s^{2}}{8 M_{W}^{4}}\right) \\
& +\frac{L n(s)}{\sqrt{\left(s-4 m_{\tilde{\tau}}^{2}\right)\left(s-4 M_{W}^{2}\right)}\left(m_{\tilde{\nu}}^{2}+3 m_{\tilde{\tau}}^{2}-2 M_{W}^{2}\right.} \\
& -\frac{1}{2} \frac{\left(2 m_{\tilde{\tau}}^{2}+2 m_{\tilde{\nu}}^{2}-3 M_{W}^{2}\right)^{2}}{s+2\left(m_{\tilde{\nu}}^{2}-m_{\tilde{\tau}}^{2}-M_{W}^{2}\right)}-\frac{\left(m_{\tilde{\nu}}^{2}-m_{\tilde{\tau}}^{2}\right)^{2}}{M_{W}^{2}} \frac{2 m_{\tilde{\tau}}^{2}+2 m_{\tilde{\nu}}^{2}-3 M_{W}^{2}}{s+2\left(m_{\tilde{\nu}}^{2}-m_{\tilde{\tau}}^{2}-M_{W}^{2}\right)} \\
& +\frac{\left(m_{\tilde{\nu}}^{2}-m_{\tilde{\tau}}^{2}\right)^{3}}{M_{W}^{4}}\left(1-\frac{1}{4} \frac{m_{\tilde{\nu}}^{2}-m_{\tilde{\tau}}^{2}}{s+2\left(m_{\tilde{\nu}}^{2}-m_{\tilde{\tau}}^{2}-M_{W}^{2}\right)}\right) \\
& +K_{H}(s)\left(\frac{s}{2}-2 m_{\tilde{\nu}}^{2}-2 m_{\tilde{\tau}}^{2}+M_{W}^{2}-\frac{s\left(m_{\tilde{\nu}}^{2}-m_{\tilde{\tau}}^{2}\right)}{M_{W}^{2}}\right. \\
& \left.\left.-3 \frac{\left(m_{\tilde{\nu}}^{2}-m_{\tilde{\tau}}^{2}\right)^{2}}{M_{W}^{2}}\left(1-\frac{s}{2 M_{W}^{2}}\right)\right)\right) \\
& =
\end{aligned}
$$

where

$$
\operatorname{Ln}(s)=\ln \left[\frac{s+2\left(m_{\tilde{\nu}}^{2}-m_{\tilde{\tau}}^{2}-M_{W}^{2}\right)-\sqrt{\left(s-4 m_{\tilde{\tau}}^{2}\right)\left(s-4 M_{W}^{2}\right)}}{s+2\left(m_{\tilde{\nu}}^{2}-m_{\tilde{\tau}}^{2}-M_{W}^{2}\right)+\sqrt{\left(s-4 m_{\tilde{\tau}}^{2}\right)\left(s-4 M_{W}^{2}\right)}}\right]
$$

The antisymmetric part gives instead:

$$
\begin{aligned}
\sigma_{\text {asym }}(s)= & \frac{g_{2}^{4} \cos ^{4} \theta_{\tilde{\tau}}}{32 \pi\left(s-4 m_{\tilde{\tau}}^{2}\right)} \sqrt{\left(1-\frac{4 m_{\tilde{\tau}}^{2}}{s}\right)\left(1-\frac{4 M_{W}^{2}}{s}\right)} \times \\
& \times\left[1+\left(1-G_{Z}(s)\right) \frac{s-2 m_{\tilde{\nu}}^{2}+2 m_{\tilde{\tau}}^{2}+2 M_{W}^{2}}{s-M_{Z}^{2}}\right. \\
& -\left(1-G_{Z}(s)\right)^{2} \frac{\left(s-4 m_{\tilde{\tau}}^{2}\right)\left(2 s+M_{W}^{2}\right)}{\left(s-M_{Z}^{2}\right)^{2}} \\
& +\frac{M_{W}^{2}\left(s-4 m_{\tilde{\tau}}^{2}\right)}{\left(s-M_{Z}^{2}\right)^{2}}\left(\frac{M_{Z}^{2}}{M_{W}^{2}}-1-G_{Z}(s)\left(\frac{s}{M_{W}^{2}}-1\right)\right)^{2} \\
& +2 \frac{m_{\tilde{\nu}}^{2}-m_{\tilde{\tau}}^{2}}{s-M_{Z}^{2}}\left(\frac{M_{Z}^{2}}{M_{W}^{2}}-1-G_{Z}(s)\left(\frac{s}{M_{W}^{2}}-1\right)\right)
\end{aligned}
$$




$$
\begin{aligned}
& +2 \frac{\left(m_{\tilde{\nu}}^{2}-m_{\tilde{\tau}}^{2}\right)^{2}}{M_{W}^{2}\left(s-M_{Z}^{2}\right)}\left(1-G_{Z}(s)\right) \\
& -\frac{\left(m_{\tilde{\nu}}^{2}-m_{\tilde{\tau}}^{2}\right)^{2}}{M_{W}^{2}\left(s-M_{Z}^{2}\right)}\left(\frac{M_{Z}^{2}}{M_{W}^{2}}-G_{Z}(s) \frac{s}{M_{W}^{2}}\right) \\
& +\frac{\left(m_{\tilde{\nu}}^{2}+m_{\tilde{\tau}}^{2}-M_{W}^{2} / 2\right)^{2}}{m_{\tilde{\nu}}^{2}\left(s+m_{\tilde{\nu}}^{2}-2 m_{\tilde{\tau}}^{2}-2 M_{W}^{2}\right)+\left(m_{\tilde{\tau}}^{2}-M_{W}^{2}\right)^{2}} \times \\
& \times\left(1-\frac{\left(m_{\tilde{\nu}}^{2}-m_{\tilde{\tau}}^{2}\right)^{2}}{M_{W}^{2}\left(2 m_{\tilde{\nu}}^{2}+2 m_{\tilde{\tau}}^{2}-M_{W}^{2}\right)}\right)^{2} \\
& +\frac{\left(s-4 m_{\tilde{\tau}}^{2}\right)\left(s-4 M_{W}^{2}\right)}{24\left(s-M_{Z}^{2}\right)^{2}}\left(10\left(1-G_{Z}(s)\right)^{2}+\frac{M_{Z}^{4}}{M_{W}^{4}}\left(1-G_{Z}(s) \frac{s}{M_{Z}^{2}}\right)^{2}\right. \\
& \left.-4\left(1-G_{Z}(s)\right)\left(\frac{M_{Z}^{2}}{M_{W}^{2}}-\frac{1}{2}-G_{Z}(s)\left(\frac{s}{M_{W}^{2}}-\frac{1}{2}\right)\right)\right) \\
& +\frac{1}{2} \frac{\operatorname{Ln}(s)}{\sqrt{\left(s-4 m_{\tilde{\tau}}^{2}\right)\left(s-4 M_{W}^{2}\right)}}\left(\frac{\left(s-4 m_{\tilde{\tau}}^{2}+M_{W}^{2}\right)^{2}}{s+2\left(m_{\tilde{\nu}}^{2}-m_{\tilde{\tau}}^{2}-M_{W}^{2}\right)}+4\left(m_{\tilde{\nu}}^{2}+m_{\tilde{\tau}}^{2}\right)\right. \\
& -2 M_{W}^{2}-\left(1-G_{Z}(s)\right) \frac{s\left(s-20 m_{\tilde{\tau}}^{2}+8 M_{W}^{2}\right)}{s-M_{Z}^{2}} \\
& -4\left(1-G_{Z}(s)\right) \frac{M_{W}^{2}\left(4 m_{\tilde{\tau}}^{2}-M_{W}^{2}\right)+m_{\tilde{\nu}}^{2}\left(m_{\tilde{\nu}}^{2}-m_{\tilde{\tau}}^{2}\right)}{s-M_{Z}^{2}} \\
& -2 \frac{m_{\tilde{\nu}}^{2}-m_{\tilde{\tau}}^{2}}{s-M_{Z}^{2}}\left(\frac{M_{Z}^{2}}{M_{W}^{2}}-1-G_{Z}(s)\left(\frac{s}{M_{W}^{2}}-1\right)\right)\left(s-2 m_{\tilde{\nu}}^{2}-6 m_{\tilde{\tau}}^{2}+2 M_{W}^{2}\right) \\
& +2 \frac{\left(m_{\tilde{\nu}}^{2}-m_{\tilde{\tau}}^{2}\right)^{2}}{M_{W}^{2}\left(s-M_{Z}^{2}\right)}\left(1-G_{Z}(s)\right)\left(s+2 m_{\tilde{\nu}}^{2}-2 m_{\tilde{\tau}}^{2}-2 M_{W}^{2}\right) \\
& -2 \frac{\left(m_{\tilde{\nu}}^{2}-m_{\tilde{\tau}}^{2}\right)^{2}}{M_{W}^{2}}\left(1+\frac{s-4 m_{\tilde{\tau}}^{2}+M_{W}^{2}}{s+2\left(m_{\tilde{\nu}}^{2}-m_{\tilde{\tau}}^{2}-M_{W}^{2}\right)}\right) \\
& -\frac{\left(m_{\tilde{\nu}}^{2}-m_{\tilde{\tau}}^{2}\right)^{2}}{M_{W}^{2}} \frac{s+2\left(m_{\tilde{\nu}}^{2}-m_{\tilde{\tau}}^{2}-M_{W}^{2}\right)}{s-M_{Z}^{2}}\left(\frac{M_{Z}^{2}}{M_{W}^{2}}-G_{Z}(s) \frac{s}{M_{W}^{2}}\right) \\
& \left.\left.+\frac{\left(m_{\tilde{\nu}}^{2}-m_{\tilde{\tau}}^{2}\right)^{4}}{M_{W}^{4}\left(s+2\left(m_{\tilde{\nu}}^{2}-m_{\tilde{\tau}}^{2}-M_{W}^{2}\right)\right)}\right)\right] .
\end{aligned}
$$

\section{References}

[1] For a compilation of bounds on charged or coloured relics, see the Particle Data Group, W.-M. Yao et al., J. Phys. G 33, 1 (2006)

[2] L. Covi, J. E. Kim and L. Roszkowski, Phys. Rev. Lett. 82 (1999) 4180 arXiv:hep-ph/9905212]; L. Covi, H. B. Kim, J. E. Kim and L. Roszkowski, JHEP 0105 (2001) 033 [arXiv:hep-ph/0101009]; L. Covi, L. Roszkowski, R. Ruiz de Austri and M. Small, JHEP 0406 (2004) 003 [arXiv:hep-ph/0402240].

[3] L. Covi, L. Roszkowski and M. Small, JHEP $0207 \quad$ (2002) 023 arXiv:hep-ph/0206119. 
[4] A. Brandenburg and F. D. Steffen, JCAP 0408 (2004) 008 [arXiv:hep-ph/0405158]. K. Y. Choi, L. Roszkowski and R. Ruiz de Austri, JHEP 0804 (2008) 016 arXiv:0710.3349 [hep-ph]]. H. Baer and H. Summy, arXiv:0803.0510 [hep-ph].

[5] J. L. Feng, A. Rajaraman and F. Takayama, Phys. Rev. Lett. 91 (2003) 011302 arXiv:hep-ph/0302215|; J. R. Ellis, K. A. Olive, Y. Santoso and V. C. Spanos, Phys. Lett. B 588 (2004) 7 arXiv:hep-ph/0312262 ; J. L. Feng, S. f. Su and F. Takayama, Phys. Rev. D 70 (2004) 063514 [arXiv:hep-ph/0404198]; D. G. Cerdeno, K. Y. Choi, K. Jedamzik, L. Roszkowski and R. Ruiz de Austri, JCAP 0606 (2006) 005 arXiv:hep-ph/0509275. J. L. Feng, B. T. Smith and F. Takayama, Phys. Rev. Lett. 100 (2008) 021302 [arXiv:0709.0297 [hep-ph]].

[6] J. L. Feng, S. f. Su and F. Takayama, Phys. Rev. D 70 (2004) 075019 arXiv:hep-ph/0404231]; L. Roszkowski, R. Ruiz de Austri and K. Y. Choi, JHEP 0508 (2005) 080 [arXiv:hep-ph/0408227].

[7] W. Buchmuller, K. Hamaguchi, M. Ratz and T. Yanagida, Phys. Lett. B 588 (2004) 90 arXiv:hep-ph/0402179]; K. Hamaguchi, Y. Kuno, T. Nakaya and M. M. Nojiri, Phys. Rev. D 70 (2004) 115007 arXiv:hep-ph/0409248|; J. L. Feng and B. T. Smith, Phys. Rev. D 71 (2005) 015004 [Erratum-ibid. D 71 (2005) 0109904] [arXiv:hep-ph/0409278]; H. U. Martyn, Eur. Phys. J. C 48 (2006) 15 arXiv:hep-ph/0605257]; J. R. Ellis, A. R. Raklev and O. K. Oye, JHEP 0610 (2006) 061 [arXiv:hep-ph/0607261]; K. Hamaguchi, M. M. Nojiri and A. de Roeck, JHEP 0703 (2007) 046 [arXiv:hep-ph/0612060].

[8] M. Fairbairn, A. C. Kraan, D. A. Milstead, T. Sjostrand, P. Skands and T. Sloan, Phys. Rept. 438 (2007) 1 arXiv:hep-ph/0611040|.

[9] See e.g. the review by B. Fields and S. Sarkar, in W. M. Yao et al. [Particle Data Group], J. Phys. G 33 (2006) 1 arXiv:astro-ph/0601514.

[10] D. Lindley, Astrophys. J. 294 (1985) 1; M. H. Reno and D. Seckel, Phys. Rev. D 37 (1988) 3441; S. Dimopoulos, R. Esmailzadeh, L. J. Hall and G. D. Starkman, Astrophys. J. 330 (1988) 545; R. J. Scherrer and M. S. Turner, Astrophys. J. 331 (1988) 19 [Astrophys. J. 331 (1988) 33]; J. R. Ellis, G. B. Gelmini, J. L. Lopez, D. V. Nanopoulos and S. Sarkar, Nucl. Phys. B 373 (1992) 399.

[11] M. Pospelov, Phys. Rev. Lett. 98 (2007) 231301 [arXiv:hep-ph/0605215]; K. Kohri and F. Takayama, Phys. Rev. D 76 (2007) 063507 [arXiv:hep-ph/0605243|; M. Kaplinghat and A. Rajaraman, Phys. Rev. D $\mathbf{7 4}$ (2006) 103004 arXiv:astro-ph/0606209 .

[12] R. H. Cyburt, J. R. Ellis, B. D. Fields, K. A. Olive and V. C. Spanos, JCAP 0611 (2006) 014 arXiv:astro-ph/0608562]; J. Pradler and F. D. Steffen, Phys. Lett. B 648 (2007) 224 |arXiv:hep-ph/0612291|; M. Kawasaki, K. Kohri and T. Moroi, Phys. Lett. B 649 (2007) 436 [arXiv:hep-ph/0703122]; J. Pradler and F. D. Steffen, arXiv:0710.2213 [hep-ph]; J. Kersten and K. Schmidt-Hoberg, JCAP 0801 (2008) 011 arXiv:0710.4528 [hep-ph]]; F. D. Steffen, arXiv:0806.3266 [hep-ph].

[13] S. Wolfram, Phys. Lett. B82 (1979) 65; 
[14] E. Nardi and E. Roulet, Phys. Lett. B 245 (1990) 105.

[15] K. Griest and M. Kamionkowski, Phys. Rev. Lett. 64 (1990) 615.

[16] M. Cirelli, A. Strumia and M. Tamburini, Nucl. Phys. B 787 (2007) 152 arXiv:0706.4071 [hep-ph]];

[17] A. Sommerfeld, Atombau und Spektrallinien, Band 2, Vieweg \& Sohn (1939);

A. D. Sakharov, Zh. Eksp. Teor. Fiz. 18, 631 (1948) [Sov. Phys. Usp. 34, 375 (1991)];

J. S. Schwinger, Particles, sources, and fields. Vol. 2, Addison-Wesley (1989) (Advanced book classics series).

[18] A. Arvanitaki, C. Davis, P. W. Graham, A. Pierce and J. G. Wacker, Phys. Rev. D 72 (2005) 075011 arXiv:hep-ph/0504210.

[19] J. Hisano, S. Matsumoto, M. Nagai, O. Saito and M. Senami, Phys. Lett. B 646 (2007) 34 arXiv:hep-ph/0610249.

[20] A. Freitas, Phys. Lett. B 652 (2007) 280 arXiv:0705.4027 [hep-ph]];

[21] N. Baro, F. Boudjema and A. Semenov, Phys. Lett. B 660 (2008) 550 arXiv:0710.1821 [hep-ph]]; J. March-Russell, S. M. West, D. Cumberbatch and D. Hooper, arXiv:0801.3440 [hep-ph].

[22] A. Strumia, arXiv:0806.1630 [hep-ph].

[23] E. W. Kolb and M. S. Turner, The Early Universe, Front. Phys. 69 (1990) 1.

[24] P. Gondolo and G. Gelmini, Nucl. Phys. B 360 (1991) 145.

[25] T. Asaka, K. Hamaguchi and K. Suzuki, Phys. Lett. B 490 (2000) 136 arXiv:hep-ph/0005136.

[26] W. Beenakker, R. Hopker, M. Spira and P. M. Zerwas, Nucl. Phys. B 492, 51 (1997) arXiv:hep-ph/9610490.

[27] S. Raby, Phys. Lett. B 422 (1998) 158 arXiv:hep-ph/9712254]; H. Baer, K. m. Cheung and J. F. Gunion, Phys. Rev. D 59 (1999) 075002 |arXiv:hep-ph/9806361.

[28] A. H. Hoang, Phys. Rev. D 56, 7276 (1997) arXiv:hep-ph/9703404); A. H. Hoang, A. V. Manohar, I. W. Stewart and T. Teubner, Phys. Rev. D 65, 014014 (2002) arXiv:hep-ph/0107144.

[29] J. Kublbeck, H. Eck and R. Mertig, Nucl. Phys. Proc. Suppl. 29A, 204 (1992).

[30] S. J. J. Gates and O. Lebedev, Phys. Lett. B 477 (2000) 216 [arXiv:hep-ph/9912362].

[31] J. Kang, M. A. Luty and S. Nasri, arXiv:hep-ph/0611322.

[32] J. Dunkley et al. [WMAP Collaboration], arXiv:0803.0586 [astro-ph].

[33] See R. Lamon and R. Durrer, Phys. Rev. D 73 (2006) 023507 arXiv:hep-ph/0506229 and references therein. 
[34] See K. Abazajian, G. M. Fuller and W. H. Tucker, Astrophys. J. 562 (2001) 593 arXiv:astro-ph/0106002; A. Boyarsky, A. Neronov, O. Ruchayskiy and M. Shaposhnikov, Mon. Not. Roy. Astron. Soc. 370 (2006) 213 arXiv:astro-ph/0512509; G. Bertone, W. Buchmuller, L. Covi and A. Ibarra, JCAP 0711 (2007) 003 arXiv:0709.2299 [astro-ph]]; H. Yuksel and M. D. Kistler, Phys. Rev. D 78 (2008) 023502 arXiv:0711.2906 [astro-ph]] and references therein.

[35] T. Yamagata, Y. Takamori and H. Utsunomiya, Phys. Rev. D 47 (1993) 1231.

[36] P. F. Smith, J. R. J. Bennett, G. J. Homer, J. D. Lewin, H. E. Walford and W. A. Smith, Nucl. Phys. B 206 (1982) 333.

[37] T. K. Hemmick et al., Phys. Rev. D 41 (1990) 2074.

[38] P. Verkerk, G. Grynberg, B. Pichard, M. Spiro, S. Zylberajch, M. E. Goldberg and P. Fayet, Phys. Rev. Lett. 68 (1992) 1116.

[39] E. B. Norman, R. B. Chadwick, K. T. Lesko, R. M. Larimer and D. C. Hoffman, Phys. Rev. D 39 (1989) 2499.

[40] M. Kawasaki, K. Kohri and T. Moroi, Phys. Rev. D 71 (2005) 083502 arXiv:astro-ph/0408426.

[41] K. Jedamzik, Phys. Rev. D 74 (2006) 103509 arXiv:hep-ph/0604251.

[42] M. Kawasaki, K. Kohri and T. Moroi, Phys. Lett. B 649 (2007) 436 arXiv:hep-ph/0703122; M. Kawasaki, K. Kohri, T. Moroi and A. Yotsuyanagi, arXiv:0804.3745 [hep-ph].

[43] C. Bird, K. Koopmans and M. Pospelov, arXiv:hep-ph/0703096; T. Jittoh, K. Kohri, M. Koike, J. Sato, T. Shimomura and M. Yamanaka, Phys. Rev. D 76 (2007) 125023 arXiv:0704.2914 [hep-ph]]; K. Jedamzik, Phys. Rev. D 77 (2008) 063524 arXiv:0707.2070 [astro-ph]]. T. Jittoh, K. Kohri, M. Koike, J. Sato, T. Shimomura and M. Yamanaka, arXiv:0805.3389 [hep-ph]; D. Cumberbatch, K. Ichikawa, M. Kawasaki, K. Kohri, J. Silk and G. D. Starkman, Phys. Rev. D 76 (2007) 123005 arXiv:0708.0095 [astro-ph]]; M. Kusakabe, T. Kajino, R. N. Boyd, T. Yoshida and G. J. Mathews, Phys. Rev. D 76 (2007) 121302 [arXiv:0711.3854 [astro-ph]].

[44] K. Jedamzik, JCAP 0803 (2008) 008 [arXiv:0710.5153 [hep-ph]].

[45] K. Hamaguchi, T. Hatsuda, M. Kamimura, Y. Kino and T. T. Yanagida, Phys. Lett. B 650 (2007) 268 arXiv:hep-ph/0702274.

[46] J. Pradler and F. D. Steffen, arXiv:0710.2213 [hep-ph].

[47] G. Belanger, F. Boudjema, A. Pukhov and A. Semenov, Comput. Phys. Commun. 149 (2002) 103 arXiv:hep-ph/0112278,, Comput. Phys. Commun. 174 (2006) 577 arXiv:hep-ph/0405253|, Comput. Phys. Commun. 176 (2007) 367 [arXiv:hep-ph/0607059].

[48] F. D. Steffen, JCAP 0609 (2006) 001 arXiv:hep-ph/0605306. 
[49] A. Brandenburg, L. Covi, K. Hamaguchi, L. Roszkowski and F. D. Steffen, Phys. Lett. B 617 (2005) 99 arXiv:hep-ph/0501287.

[50] LEPSUSYWG, ALEPH, DELPHI, L3 and OPAL experiments, note LEPSUSYWG/02-05.1 (http://lepsusy.web.cern.ch/lepsusy/Welcome.html).

[51] J. L. Diaz-Cruz, J. R. Ellis, K. A. Olive and Y. Santoso, JHEP 0705 (2007) 003 arXiv:hep-ph/0701229].

[52] J. Nachtman, talk at the symposium "The Hunt for Dark Matter", Fermilab, 10-12 May 2007.

[53] F. Takayama and M. Yamaguchi, Phys. Lett. B 485 (2000) 388 arXiv:hep-ph/0005214; ; W. Buchmuller, L. Covi, K. Hamaguchi, A. Ibarra and T. Yanagida, JHEP 0703 (2007) 037 [arXiv:hep-ph/0702184]. 